\title{
WestVirginiaUniversity
}

THE RESEARCH REPOSITORY @ WVU

Graduate Theses, Dissertations, and Problem Reports

2013

\section{Distributed Joint Sensor and Target Location Estimation}

Muammar Ali

West Virginia University

Follow this and additional works at: https://researchrepository.wvu.edu/etd

\section{Recommended Citation}

Ali, Muammar, "Distributed Joint Sensor and Target Location Estimation" (2013). Graduate Theses, Dissertations, and Problem Reports. 3605.

https://researchrepository.wvu.edu/etd/3605

This Thesis is protected by copyright and/or related rights. It has been brought to you by the The Research Repository @ WVU with permission from the rights-holder(s). You are free to use this Thesis in any way that is permitted by the copyright and related rights legislation that applies to your use. For other uses you must obtain permission from the rights-holder(s) directly, unless additional rights are indicated by a Creative Commons license in the record and/ or on the work itself. This Thesis has been accepted for inclusion in WVU Graduate Theses, Dissertations, and Problem Reports collection by an authorized administrator of The Research Repository @ WVU. For more information, please contact researchrepository@mail.wvu.edu. 


\title{
Distributed Joint Sensor and Target Location Estimation
}

\author{
by \\ Muammar Ali \\ Thesis submitted to the \\ Benjamin M. Statler College of Engineering And Mineral Resources \\ at West Virginia University \\ in partial fulfillment of the requirements \\ for the degree of \\ Master of Science \\ in \\ Electrical Engineering \\ Brian D. Woerner, Ph.D. \\ Matthew C. Valenti, Ph.D. \\ Natalia A. Schmid, D.Sc., Chair \\ Lane Department of Computer Science and Electrical Engineering \\ Morgantown, West Virginia \\ 2013
}

Keywords: maximum likelihood estimation, wireless sensor network, Bisection Algorithm, target localization

Copyright 2013 Muammar Ali 


\author{
Abstract \\ Distributed Joint Sensor and Target Location Estimation \\ by \\ Muammar Ali \\ Master of Science in Electrical Engineering \\ West Virginia University \\ Natalia A. Schmid, D.Sc., Chair
}

The area of wireless sensor networks (WSN) is a fast growing area and has a variety of applications such as environmental monitoring, health care, agricultural applications, and military applications. While this research area has been active since 1980s, new applications bring new constraints, and these lead to new open problems. This thesis approaches one of them. It focuses on the problem of target location estimation with limited knowledge about sensor locations in a WSN. Sensors in a WSN are randomly deployed over a known area. The true locations of the sensors are unknown, but each sensor node is equipped with positioning technolgy system such as GPS generating noisy measurements of the true unknown location. A target is modeled as a point source generating a spatial parametric signal or field. The shape of the parametric field is known, but the location of the point source is unknown. The sensors monitor the field and report their noisy measurements to a base station called Fusion Center (FC). The measurements are wirelessly transmitted to the FC via Additive White Gaussian Noise (AWGN) channels. We assume two cases of transmission channel in this thesis. In the first case, the observed data are modulated using a linear analog modulation such as amplitude. In the second case, the observed data are quantized to $\mathrm{M}$ quantization levels and then transmitted to the FC using a digital modulation scheme such as ON-OFF Keying (OOK). Each sensor transmits two sets of data: (1) noisy measurements of the field and (2) the measurements of the sensor position provided by its positioning system. Given noisy measurements of the field and noisy measurements of sensor locations, the task of the FC is to find the location of the point source. The FC applies the Maximum Likelihood estimation approach to solve for unknown parameters. In this thesis, the numerical solution is due to the Bisection method that iteratively estimates the location of the point source and the location of sensors and alternates these two steps. The field generated by a point source is modeled as a Gaussian bell function. The measurements of sensor location are assumed to be drawn from a two-dimensional Gaussian distribution with the true location of a sensor as its mean and known covariance matrix. The performance of the proposed solution is measured in terms of the square error (SE) between the true and estimated location of the point source. The MS is analyzed as a function of many parameters of the WSN, FC and parametric field. A comparison with a baseline case, when the locations of sensors are known to the FC, is made. 


\section{Acknowledgements}

I would like to express my sincere gratitude and deepest appreciation to Dr. Natalia A. Schmid for her constant support during my thesis. Her patience helped me a lot to overcome many obstacles during the thesis. I am also thankful to her for helping me during writing the thesis.

I would also like to thank Dr. Brian Woerner and Dr. Matthew C. Valenti being members of my committee, and I would like to thank them for the time that they will take to review and comment on my thesis.

Special thanks go to my friend Marwan Alkhweldi for his valuable support and feedback that helped me to understand my research better. I also would like to thank my friends Fathi Masoud and Ahmed Ammar for many useful discussions. .

Last but not the least, I would like to express my unbounded gratitude to my parents and my wife for their unconditional support and encouragement throughout my life. 


\section{Contents}

$\begin{array}{ll}\text { Acknowledgements } & \text { iii }\end{array}$

List of Figures $\quad$ vi

Notation $\quad$ vii

1 Introduction $\quad 1$

2 Literature Review $\quad 5$

3 Problem Statement and Proposed Solution $\quad 10$

3.1 Models and Assumptions . . . . . . . . . . . . . . . . . . . . 11

3.1 .1 Analog Modulation . . . . . . . . . . . . . . . 12

3.1 .2 Digital Modulation . . . . . . . . . . . . . . 15

3.1.3 Justification of the Model for Transmission Channels . . . . . . . . . 18

3.2 Proposed Solution . . . . . . . . . . . . . . . . . . 21

3.2.1 Maximum Likelihood Solution for Analog Case . . . . . . . . . . . . 21

3.2.2 Maximum Likelihood Solution for Digital Case . . . . . . . . . . . . 23

3.2 .3 Iterative Solution . . . . . . . . . . . . . . . . . 25

$4 \quad$ Numerical Results $\quad 27$

4.1 Auxiliary Performance Measures . . . . . . . . . . . . . . . . . . . . 28

4.1 .1 Probability of Outliers . . . . . . . . . . . . . . 28

4.1.2 Signal to Noise Ratio for Analog Channel . . . . . . . . . . . . . . . . 28

4.1.3 Signal to Noise Ratio for Digital Channel . . . . . . . . . . . . . . . . 29

4.2 Numerical Results for Analog Case . . . . . . . . . . . . . . . . . . 30

4.2.1 The Effect of Varying the Number of Sensors . . . . . . . . . . . . . . 30

4.2 .2 Varying the Strength of the Field $\Lambda \ldots \ldots$. . . . . . . . . 30

4.2.3 The Probability of Outliers with Varying the Number of Sensors . . 31

4.2.4 Comparison with the Baseline Case when Sensor Positions are Known 31

4.2 .5 Varying Signal to Noise Ratio . . . . . . . . . . . . . . . . . . . . 33

4.2.6 Sensitivity of the Algorithm to the Choice of Initial Values . . . . . . 35

4.3 Numerical Results for Digital Case . . . . . . . . . . . . . . . . . 36

4.3.1 The Effect of Varying the Number of Sensors . . . . . . . . . . . . . . . . 36

4.3 .2 Varying the Strength of the Field $\Lambda \ldots \ldots$. . . . . . . . 37 
4.3.3 Probability of Outliers with Varying the Number of Sensors . . . . . 38

4.3.4 Comparison with the Case when Sensor Locations are known . . . . 38

4.3 .5 Varying Signal to Noise Ratio . . . . . . . . . . . . . . . . . . 39

4.3.6 Varying the Number of Quantization Levels . . . . . . . . . . . . . 42

4.3.7 Sensitivity of the Algorithm to the Choice of Initial Values . . . . . . 42

5 Conclusion and Future Work 44

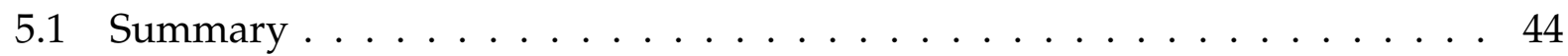

5.2 Claimed Novelties . . . . . . . . . . . . . . . . . . 45

5.3 Future Research . . . . . . . . . . . . . . . . . . . . 45

$\begin{array}{ll}\text { References } & 47\end{array}$ 


\section{List of Figures}

1.1 Components of Sensor Node. . . . . . . . . . . . . . . . . . 2

3.1 Signal Processing using Analog Modulation. . . . . . . . . . . . . . . . . 13

3.2 Signal processing for digital modulation and demodulation. . . . . . . . . . 20

3.3 Flow chart of the bisection algorithm. . . . . . . . . . . . . 26

4.1 Dependence of the simulated SE on the number of sensors. . . . . . . . . . . 31

4.2 Dependence of the simulated SE on the strength of the field. . . . . . . . . . 32

4.3 Dependence of the probability of outliers on the threshold $\tau$ parametrized by three different values of K. . . . . . . . . . . . . . . . . 32

4.4 The performance comparison between estimators with certainty and uncertainty in sensor locations. The network is composed of $K=50$ sensors. . 33

4.5 Dependence of the simulated SE on the signal to noise ratio. . . . . . . . . . 34

4.6 Dependence of the probability of outliers on the value of $\tau$. The plots are parametrized by three different values of SNR . . . . . . . . . . . . . . 34

4.7 Dependence of estimating sensor locations on the signal to noise ratio. . . . 35

4.8 The effect of choosing the initial values on the performance of the estimator. 36

4.9 Dependence of the simulated SE on the number of sensors for the case of the digital channel with $M=8 \ldots \ldots \ldots$. . . . . . . . . . . 37

4.10 Dependence of the simulated SE on the strength of the field in the case of the digital channel with $M=8 \ldots \ldots \ldots \ldots$

4.11 Dependence of the probability of outliers on the threshold $\tau$ for three choices

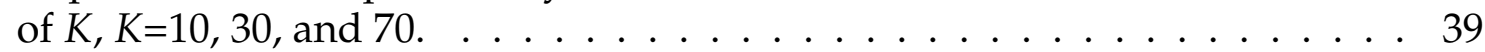

4.12 The performance comparison between estimators with certainty and uncertainty in sensor locations. The network is composed of $K=50$ sensors. . 39

4.13 Dependence of the simulated SE on the signal to noise ratio, $S N R_{o}=S N R_{c} . \quad 40$

4.14 Dependence of the probability of outliers on the threshold values. The plots are parametrized by three different values of $S N R_{0}=S N R_{c}=10,15,20 . \quad$. . 41

4.15 Dependence of estimating sensor locations on the signal to noise ratio. . . . 41

4.16 Dependence of the simulated SE on the Number of Quantization Levels. . . 42

4.17 The effect of choosing the initial values on the performance of the estimator. 43 


\section{Notation}

We use the following notation and symbols throughout this thesis.
A area of interest
$K \quad$ number of sensors
$N \quad$ Number of sensor locations measurements per sensor
$x_{k}, y_{k} \quad$ the true locations of the k-th sensor
$x_{c}, y_{c} \quad$ object location
$\tilde{X}_{k}, \tilde{Y}_{k} \quad$ estimated sensor location of the $k-t h$ sensor via the positioning system
$G \quad$ physical field
$\theta \quad$ the unknown parameters
$W \quad$ the noise in observation channel
$T($.$) \quad the transformation of sensor measurement prior transmission$
$V \quad$ the noise in transmission channel
$R \quad$ the noisy sensor measurements of the field
$Z \quad$ the signal received by the FC
$\mathrm{RX}, \mathrm{RY}$ the noisy transformed versions of sensor location supplied by the positioning syster
$\sigma^{2} \quad$ the noise variance of field measurements in the observation channel
$\xi^{2}$ the variance of the estimated measurements of sensor positions
$\eta^{2} \quad$ the noise variance in the communication channel
$\Lambda \quad$ the signal power of the field
$M \quad$ the number of quantization levels
$\lambda \quad$ the $j-t h$ reproduction reproduction points of the quantizer
$\tau_{j} \quad$ a boundary point of the $j-$ th quantizer regions
$p_{k, j} \quad$ the probability for the output of the sensor $k$ to map to the $j$-th reproduction point
$Q(R) \quad$ quantized sensor measurements
$Q_{\tilde{X}}, Q_{\tilde{Y}} \quad$ quantized sensor position estimates
$f_{Q_{R}} \quad$ the $p d f$ of the quantized sensor measurements
$f_{Q_{\tilde{X}}}, f_{Q_{\tilde{Y}}}$ the $p d f$ of the quantized measurements of sensor locations
$f_{Z} \quad$ the $p d f$ of the noisy sensor measurements
$f_{R X}, f_{R Y}$ the $p d f$ of the quantized and transmitted measurements of sensor locations 


\section{Chapter 1}

\section{Introduction}

The area of wireless sensor networks (WSN) is a fast growing area, and has a variety of applications such as environmental monitoring [1], health care [2], agricultural applications [3], and military applications [4, 5, 6]. However, designing WSNs is a challenging task. It requires a good knowledge of wireless communication protocols, power allocation and signal processing $[7,8]$. Building WSNs assumes dealing with various constraints such as bandwidth constraints, energy constraints, and communication constraints $[9,10,11]$.

WSNs consist of spatially distributed nodes. A sensor node is composed of four basic components: sensing unit, processing unit (Micro-controller or Embedded Computing System)[12], transceiver unit and power unit [11, 13]. Sensors are a type of transducers, devices that convert one form of energy into another [11, 14].

The first component of a sensor node is a sensory unit, which senses a process and gathers information about a certain phenomenon and converts measurements into a form that can be transmitted to neighboring nodes or to a base station. Sensors don't need to be very close to the object in order to gather the information. They can accumulate the data remotely via remote sensing. After collecting the desired data, they manipulate them in a way that corresponds to the hardware and the software structure of the system. The second component is the processing unit, which performs some operations on a sensory data, for instance, amplification, attenuation or removing the undesirable noise. The third component is a transceiver [15], which connects the node to the network. The fourth 


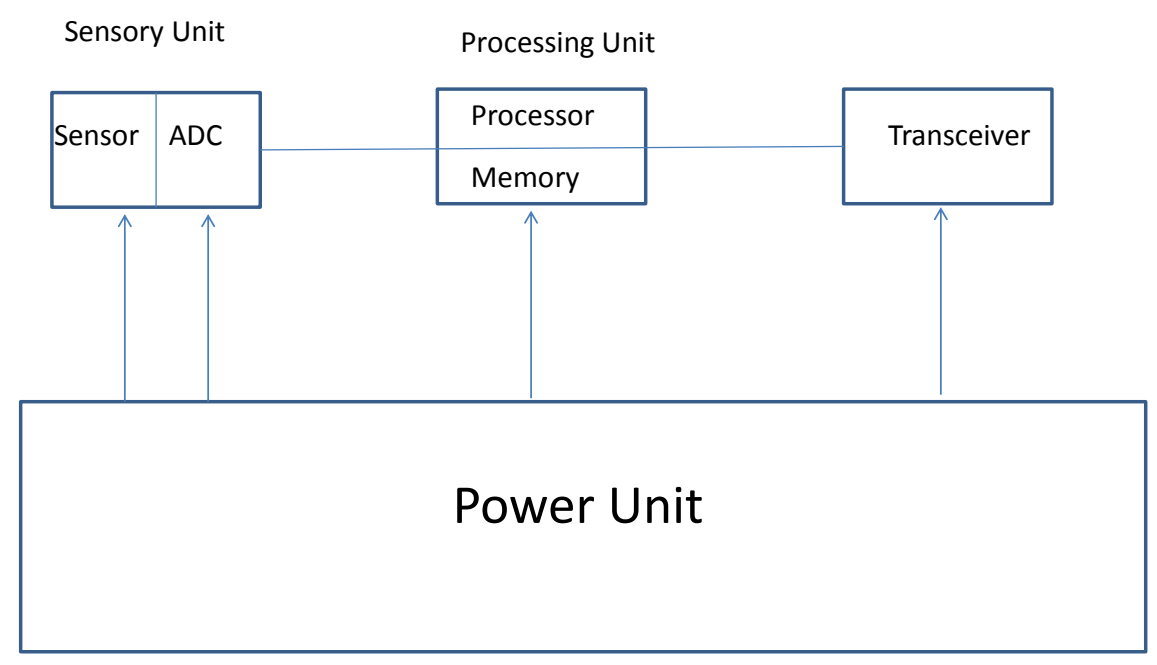

Figure 1.1: Components of Sensor Node.

component is a power unit, which powers all the components in a sensor node [11]. The four mentioned components of the sensor node are shown in Fig. 1.1.

There are two main types of configurations in WSNs. In the first type of WSN, sensors send their real measurements to a base station also known as Fusion Center (FC) for joint processing and making an inference. This type of networks are known in literature as distributed sensors networks. The second type is called decentralized sensor networks. In this type, sensors exchange information with their neighbors and perform local inferences [16].

WSNs are built to incorporate multiple constraints. One of these constraints is the energy constraint, which is due to the limited energy stored in the sensors batteries. Therefore, preserving the energy and wisely distributing it among sensors is one of the major factors to consider while designing wireless sensor networks. In the past two decades, many methods to save the energy and optimally allocate it across the network of sensors have been proposed. One of the proposed techniques is to limit the communication process between the sensory unit and the base station unless there is important data to be send $[10,11]$.

Another constraint is a communication constraint. For some applications the band- 
width that can be utilized by a system is limited. Therefore, to fulfill this requirement, a variety of algorithms managing bandwidth constraint were introduced. Many of these algorithms focused on minimizing the amount of data to be sent to the base station. This is typically done by giving priorities to certain nodes to send their data[9].

As stated above, WSNs are used for a variety of applications such as source localization, signal detection and object tracking applications [7, 8]. Many researchers assume that all parameters of WSN are known or well estimated. However, in practice this may not be the case.

In this thesis, we develop a framework for distributed estimation of location of a source in the presence of the uncertainty in sensor locations. The problem of sensor localization ( determining positions of sensors in a WSN ) is often considered separately from the inference problem. It is often assumed that sensors are placed on the ground and they determine their position immediately, at the time of their placement.

WSN localization is very important in many applications of WSN, however, it makes the architecture of WSN more complicated, since more hardware components are required for localization such as node beacon (the node that knows its location), positioning system to define the location of the sensor, synchronization equipments, etc $[8,17,18]$. Consequently, network localization increases the cost of WSN, energy consumption, as well as software and hardware complexity [19]. As a separate topic, WSN localization has attracted attention of many researchers [20,21]. Many adaptive solutions have been proposed.

In this thesis, we assume only partial knowledge of positions of sensors in a network. Each sensor node is equipped with a positioning system. We also assume that the main task of the network is to localize an object (modeled as a point source radiating energy) sensed by the network.

The nodes in the network transmit both their measurements of a signal generated by the point source and the readings of the estimated sensor position to a Fusion Center. We pose the problem of source localization with uncertainty in sensor location as the problem of joint estimation of the source and sensor location. The FC implements an iterative algorithm that alternatively updates the estimates of sensor positions and the 
source position.

The remainder of this thesis is organized as follows. Chapter two presents a literature review on the topic of source localization using wireless sensor networks. Chapter three formulates the problem of joint sensor and source localization and introduce an iterative solution. Chapter four presents numerical results. Finally, we summarize the entire work and propose some ideas for the future work in chapter five. 


\section{Chapter 2}

\section{Literature Review}

WSNs have been an active area of research since 1980s. WSNs are used in different applications such as environment monitoring, medical applications, military applications, etc $[22,23,24,25]$. Many research problems related to WSNs are addressed in the literature. One of them is the problem of WSN localization. It is defined as the problem of estimating positions of sensors in a WSN at the moment of its installation [26]. This also can be viewed as the problem of network calibration. There is a vast amount of literature devoted to this topic. The latest trend is the application of so-called consensus algorithm $[27,28,29]$ for calibration of a distributed WSN.

Another important problem relevant to the topic of this thesis is object ( target or point source) location estimation. In the past, many algorithms have been developed to estimate a target location using measurements collected by a WSN. Some of the papers considered estimation in a decentralized WSN. Others assumed a distributed set up. Many works stated the problem of object localization as a constrained optimization problem involving bandwidth and power constraints. Following, we present a brief summary of these works.

In [30], Xiao and Luo implemented a Decentralized Estimation Scheme (DES). In this scheme, they tried to minimize the utilized bandwidth and maintain the overall system performance. In order to do this, each sensor has to send a number of bits to the FC, this number of bits vary according to the signal to noise ratio (SNR) of each sensor, or the local noise variance of each sensor, which guarantees that the performance is within a con- 
stant factor of the performance achieved by the best linear unbiased estimator (BLUE)[16]. Therefore, the algorithm depends on signal to noise ratio (SNR) rather than probability density function (pdf) of the noise. SNR is calculated by each sensor and the data, which are coming from sensors, are gathered at the FC in order to estimate a real valued parameter. The algorithm achieves a low value of Mean Squared Error (MSE) with a small amount of exchanged data to satisfy the minimum bandwidth constraint. There are two considered approaches. The choice of the approach depends on whether the noise pdf is known or unknown. The numerical results show that the same MSE performance can be achieved by a universal DES with low communication requirement (measured in bits).

In [31], Wu, Huang, and Lee discuss energy-constrained decentralized best-linearunbiased estimation via partial sensor noise variance knowledge. There are two assumptions that they made on this method. The first assumption each sensor can send only quantized version of its raw data to the FC, the second assumption the sensor noise variance is unknown to the FC. Their algorithm relies on maximizing the reciprocal of MSE with the respect to noise variance distribution. They have done several approximations to derive a lower bound on the estimation of parameters to achieve a closed form solution. Their conclusion was, under budget constraints, sensors with a bad communication quality are to be turned off in order to save the designated power.

In [32], Cui, Xiao, Goldsmith, Luo, and Poor studied estimation diversity and energy efficiency in distributed sensing. In this paper, they assumed a group of sensors send their data to the FC via a fading channel, the FC uses BLUE estimator to estimate the real environment. They used joint estimation scheme under power constraint that takes in consideration channel fading and a channel noise. In addition to that, they showed that multiple sensor nodes are necessary to obtain a certain level of mixture, which will reduce the effect of channel fading during signal transmission. They came up with a result that turning off sensors with poor quality of observations will save some power and in the mean time will not affect the diversity.

In [33], Li and AlRegib studied a generic framework for energy-constrained distributed estimation in WSNs from energy-distortion perspective and introduced the concept of equivalent unit-energy MSE function. Based on this concept, two different algorithms 
were introduced according to a type of used network. For a homogeneous sensor networks, optimal energy-constrained distributed estimation algorithm was used, and for heterogeneous sensor networks, a quasi-optimal energy-constrained distributed estimation algorithm was applied. Li and AlRegib compromised between the number of active sensors and the allocated energy for each active sensor in heterogeneous sensor networks. The results of analysis show that the mentioned method can accomplish a substantial reduction in MSE when compared with other uniform schemes.

In [34], Li and AlRegib discussed function-based network lifetime and introduced a new concept for the network lifetime. In addition to that, the network is considered operative if it can generate an estimate satisfying the given distortion requirement. Otherwise, it is considered nonfunctional. Furthermore, they derived an upper bound on a function based network lifetime for estimation and maximized it by introducing the concept of equivalent unit-resource Mean Square Error (MSE) function.

In [35] Wang, Ishwar and Saligrama studied a general distributed field reconstruction problem using a dense network of finite bit rate. They developed a field reformation scheme based on quantized samples of observations which is interfered by zero-mean additive Gaussian noise independent across sensors. Each sensor is quantized via a binary quantizers, after a certain number of snapshots, the data will be sent to the FC to perform parameter estimation. One contribution in this paper is adding important hardware. This hardware is a noisy operational amplifier adequate for high-resolution distributed field reconstruction. Another contribution is in the overall improvement of the general distributed field reconstruction. Finally, the results show that when the noise, the arrangement of sensor location, and the sensor satisfy requirements stated in the paper, it is possible to drive MSE to zero with increasing sensor density.

In [36] Ribeiro and Giannakis studied deterministic mean-location parameter estimation when a quantized version of the original observations is available. In order to fulfill bandwidth constraints, Ribeiro and Giannakis introduced a type of Maximum Likelihood Estimator (MLE) that requires just one bit per sensor. Different values of signal to noise ratio were used (from low, medium to high). The simulation results show that at high SNR even a classic estimator requires transmitting a small number of extra bits greater 
than the MLE. Moreover, they implemented a good estimator for a total number of bits that converged to Cramer Rao Lower Bound (CRLB), which compromises between reducing the quantization step or giving more space to send independent measurement. Furthermore, they compared MLE with Quantized Sample Mean Estimator (QSME), and figured out that, at high value of SNR, even a least complex scheme performs close to optimum. Eventually, the conclusion was that the numerical maximization required by MLE can be considered as an optimization problem.

In [37], Ruixin, Chen and Varshney discussed the problem of fusing decisions transmitted over fading channels in WSNs and proposed a new likelihood ratio (LR)-based fusion rule. They showed that when the channel SNR is low, the fusion rule minimizes to a statistic rule in the structure of an equal gain combiner (EGC). Ruixin, Chen and Varshney proposed four fusion rules. The first fusion rule is the Optimal LR-Based Fusion Rule. This rule assumes CSI (Channel state information) knowledge regarding fading channel and local sensor performance. The second rule is the ChairVarshney fusion rule. This rule does not require knowledge of CSI, but it requires the knowledge of the probability of false alarm and detection. The third rule is the maximum ratio combiner (MRC) fusion rule. This rule requires the knowledge of the channel gain, but it does not require the knowledge of the probability of false alarm and detection. The fourth rule is the EGC fusion rule, which requires a minimum amount of information. At the end, they compared the performance of the four mentioned rules and concluded that the ChairVarshney fusion statistic rule provides a high SNR approximation to the new fusion statistic rule. The new fusion rule surpasses the EGC and the ChairVarshney fusion rule, as well it outperform the MRC fusion rule for most high values of SNR.

In [38], Ruixin and Varshney discussed the problem of target location estimation in sensor networks with quantized data. They assumed that the signal received power at the local sensors is inversely proportional to the square of distance from the target. Furthermore, they proposed an intensity based ML target location estimation method using only quantized data. In their proposed method each sensor collects data from the field, and these data are quantized to discrete values and sent to a FC. Based on the received data, the FC will locate the position of the target. Ruixin and Varshney used two dif- 
ferent estimation algorithms for target location for the purpose of comparison. The first algorithm is the MLE. In this model the estimation of the target location is determined by finding the location that maximizing the log-likelihood function of the received signal at the FC. The second algorithm is conducted by using the Weighted Average (WA) method. In this type of algorithm, the estimation of the target location is performed by taking the average weight of all sensor locations. After performing simulations using these two different methods, the results show that the ML method gives a better performance than the WA method, except when the number of sensors in the region of interest is very small. As the number of sensors increases, the performance of the ML method gets better.

In [39], Addesso, Marano, and Matta studied the estimation of target location via likelihood approximation in sensor networks . In this paper, they assumed that there is no FC, sensors communicate among each other in order to estimate the target location. Their main idea was to design a decentralized estimator through information exchanged between the nearby sensors in the network. The sensor who reaches the maximum of the likelihood will declare the position of the target in the Region Of Interest (ROI), but this sensor has to define his position before that. The main contribution of this paper is optimizing the number of communication tasks to the best number that gives the best performance. The other contribution is deriving estimated formula for the log-likelihood function when the sensor density increase without bound. Finally, they derived a formula to calculate the optimal iteration number that gives a low MSE. 


\section{Chapter 3}

\section{Problem Statement and Proposed Solution}

In this section we discuss the problem of distributed joint sensor and target location estimation and propose a solution to this problem. We assume that we have a point source generating a physical field. The field is sensed by a WSN deployed over a finite area $A$. The true locations of sensors in the WSN are unknown. However, estimates of the true sensor locations are supplied by means of a positioning technology. Each sensor takes a sample of the field generated by a point source augmented with a sample of an AWGN. Sensors send their noisy samples of the field to a FC via parallel channels with AWGN. The FC collects noisy observations from all sensors and uses the MLE rule to update the estimates of sensor locations and estimate the location of the point source. We consider two cases of local signal processing (processing of data performed at the location of each sensor). The first case assumes that sensors send their observations to the FC without any local processing. The only processing applied to the measurements is an analog modulation and demodulation. In this thesis, we assume linear modulation and synchronous demodulation. The second case assumes that the data are quantized to a number of levels and these data are sent to the FC in a digital form (as bits). This case is named as digital. The signal processing for this case assumes that sensor measurements are quantized and digitally modulated. They are then demodulated at the receiver end and used for joint parameter estimation. No decoding at the receiver end is applied. 


\subsection{Models and Assumptions}

In mathematical terms, consider a network of $K$ sensors distributed over an area $A$. The true locations of the sensors, denoted by $\left(x_{k}, y_{k}\right), k=1, \ldots . ., K$ are unknown. Sensors act independently of one another and take noisy measurements of a physical field denoted as $G(x, y),(x, y) \in A$. A sample of $G(x, y)$ at the location $\left(x_{k}, y_{k}\right)$ is denoted as $G_{k}=G\left(x_{k}, y_{k}\right)$. The field $G(x, y)$ is characterized by $L$ unknown parameters $\theta=\left[\theta_{1}, \ldots, \theta_{L}\right]^{T}$. The sensor noise, denoted by $W_{k}, k=1, \ldots K$ is known and modeled as Gaussian distributed with zero mean and variance $\sigma^{2}$. The noise of sensors is independent and identically distributed (i.i.d.). Let $R_{k}, k=1, \ldots K$ be the noisy sample of the field at the location of the $k-t h$ sensor. The $R_{k}$ is modeled as

$$
R_{k}=G_{k}+W_{k}, k=1, \ldots, K
$$

Thus, the measurements $R_{k}$ are independent Gaussian distributed with mean $G_{k}$ and variance $\sigma^{2}$.

These noisy observations are transmitted over noisy parallel channels to a FC. The method to send these observations and the required signal processing will be described below.

Denote by $T($.$) the transformation that field and sensor locations measurements un-$ dergo prior to thier transmission. Let $V_{k}$ be the AWGN in the $k$ - th transmission channel. Then, the signal received by the FC, denoted by $Z_{k}$ is modeled as:

$$
Z_{k}=T\left(R_{k}\right)+V_{k}
$$

where $V_{k}$ is $N\left(0, \eta^{2}\right)$. The sensors also have the ability to inform the FC about their positions, with an error. This error is modeled as a Gaussian random vector with zero mean and the $2 \times 2$ diagonal covariance matrix $\left[\begin{array}{cc}\xi^{2} & 0 \\ 0 & \xi^{2}\end{array}\right]$, where $\xi^{2}$ is known. This results in the following model for the uncertainty in the position of sensors:

$$
\left[\begin{array}{c}
\tilde{X}_{k} \\
\tilde{Y}_{k}
\end{array}\right] \sim N\left(\left[\begin{array}{l}
x_{k} \\
y_{k}
\end{array}\right],\left[\begin{array}{cc}
z^{2} & 0 \\
0 & z^{2}
\end{array}\right]\right)
$$


where $\left[\tilde{X}_{k}, \tilde{Y}_{k}\right]^{T}$ is the estimated measurement of the $k$ - th sensor position and $\left[x_{k}, y_{k}\right]^{T}$ is the true position, but unknown position in a vector form.

The goal of this work is to estimate a vector of unknown parameters that characterizes the deterministic field generated by a point source based on noisy observations of the underlying field. Due to the uncertainty in the sensor positions, estimates of the parameters will not be reliable. To make the estimates more reliable, the sensors positions have to be as accurate as possible. To do so, the sensors have to inform the FC about their positions as many times as the system permits, say $N$ times. To save the energy, $N$ can not be large. The model for the uncertainty in sensor positions is known to the FC. Each sensor communicates $N$ realizations of $\left[\tilde{X}_{k}, \tilde{Y}_{k}\right]^{T}$ to the FC. Denote by $\mathrm{RX}_{k}$ and $\mathrm{RY} \mathrm{Y}_{k}$ noisy transformed versions of $\tilde{X}_{k}$ and $\tilde{Y}_{k}$, respectively. Let $\left(\left(r x_{k, 1}, r y_{k, 1}\right), \ldots,\left(r x_{k, N}, r y_{k, N}\right)\right)$ be $N$ realizations of $\left(\mathrm{RX}_{k}, \mathrm{RY} \mathrm{Y}_{k}\right)$. Since we assume that the estimated measurements of the sensor locations are communicated to the FC over the same channel used to communicate noisy measurements of the field, we arrive to the following model for $\left(\mathrm{RX}_{k}, \mathrm{RY} \mathrm{Y}_{k}\right)$ :

$$
\begin{aligned}
& \mathrm{RX}_{k}=T\left(\tilde{X}_{k}\right)+\mathrm{VX}_{k}, \\
& \mathrm{RY}_{k}=T\left(\tilde{Y}_{k}\right)+\mathrm{VY}_{k},
\end{aligned}
$$

where $\mathrm{VX}_{k}$ and $V Y_{k}$ are i.i.d. Gaussian random variables. Note, $\mathrm{RX}_{k}$ and $\mathrm{RY}_{k}$ are independent random variables.

\subsubsection{Analog Modulation}

In the case when data are transmitted over analog channels, both the measurements of the field $R_{k}$ and the estimated sensor locations $\left(\left(\tilde{x}_{k, 1}, \tilde{y}_{k, 1}\right) \ldots\left(\tilde{x}_{k, N}, \tilde{y}_{k, N}\right)\right)$ are modulated to a high carrier frequency $f_{c}$ by using an amplitude modulation. In Fig. 3.1, the random variable $R_{k}$ is multiplied by $\cos \left(2 \pi f_{c} t\right)$ to form a signal $R_{k}(t)=R_{k} \cos \left(2 \pi f_{c} t\right)$. The waveform $Z_{k}(t)$ observed at the FC contains AWGN signal $V_{k}(t)$ :

$$
Z_{k}(t)=R_{k}(t)+V_{k}(t) .
$$


The power spectral density of $V_{k}(t)$ is known and equal to $N_{o} / 2$. To extract a signal space representation, the received random signal $Z_{k}(t)$ is multiplied by the same carrier signal $\cos \left(2 \pi f_{c} t\right)$ and integrated over an interval $T_{o}$ with the length $T_{o}=m / f_{c}$, where $m$ is a positive integer. The gain $2 / T_{o}$ is applied to the output signal. Given the processing steps

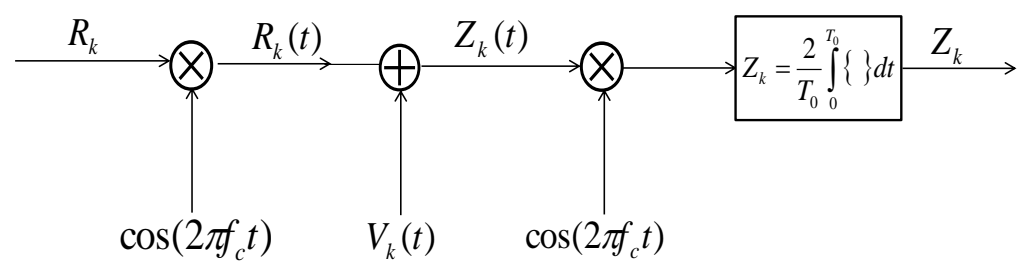

Figure 3.1: Signal Processing using Analog Modulation.

shown in Fig. 3.1, the probability density function (pdf) of the received signal can be easily evaluated. The received signal $Z_{k}$ is expressed as the following integral:

$$
Z_{k}=\frac{2}{T_{o}} \int_{0}^{T_{o}}\left\{R_{k} \cos \left(2 \pi f_{c} t\right)+V_{k}(t)\right\} \cos \left(2 \pi f_{c} t\right) d t
$$

Since $\cos ^{2}\left(2 \pi f_{c} t\right)=\frac{1}{2}+\frac{1}{2} \cos \left(4 \pi f_{c} t\right)$ and assuming that $T_{o}=m / f_{c}$,

$$
Z_{k}=R_{k}+\frac{2}{T_{o}} \sum_{i=1}^{m} \int_{(i-1) T_{\mathcal{c}}}^{i T_{c}} V_{k}(t) \cos \left(2 \pi f_{c} t\right) d t,
$$

where $T_{c}=1 / f_{c}$.

Since the second term in eq.(3.8) presents a linear combination of Gaussian random variables, the result of the integration, denote it as $V_{k}(i)$, is also a Gaussian random variable. Its mean is zero and variance is

$$
\begin{aligned}
& E\left(\int_{(i-1) T_{c}}^{i T_{c}} \int_{(i-1) T_{c}}^{i T_{c}} V_{k}(t) V_{k}(u) \cos \left(2 \pi f_{c} t\right) \cos \left(2 \pi f_{c} u\right) d t d u\right) \\
= & \int_{(i-1) T_{c}}^{i T_{c}} \int_{(i-1) T_{c}}^{i T_{c}} \frac{N_{o}}{2} \delta(t-u) \cos \left(2 \pi f_{c} t\right) \cos \left(2 \pi f_{c} u\right) d t d u \\
= & \frac{N_{o}}{2} \int_{(i-1) T_{c}}^{i T_{c}} \cos ^{2}\left(2 \pi f_{c} u\right) d u \\
= & \frac{N_{o} T_{c}}{4}
\end{aligned}
$$


and $\frac{2}{T_{o}} \sum_{i=1}^{m} V_{k}(i)$ is Gaussian distributed with zero mean and variance $\frac{4}{T_{0}^{2}}\left(\frac{N_{o} T_{c}}{4} m\right)=\frac{N_{o}}{T_{o}}$. Thus, the received random variable $Z_{k}=R_{k}+V_{k}$, where $V_{k}=\frac{2}{T_{0}} \sum_{i=1}^{m} V_{k}(i)$ is a Gaussian random variable with mean zero and variance $\frac{N_{o}}{T_{o}}$ which we will denote as $\eta^{2}$. The measurements $R_{k}$ and the additive channel noise $V_{k}$ are independent random variables.

Each sensor transmits three sequences of data to the FC. The first and the second sequences are a set of estimates of sensor locations in a shape of Gaussian random vector with the mean of the vector being the true location of sensors. The last sequence is a set of noisy field measurements at the true location of sensors. Denote by $\mathrm{RX}_{k}=$ $\left[\mathrm{RX}_{k, 1}, \ldots \mathrm{RX}_{k, N}\right]^{T}$ a vector of $N$ observations of $x$-location of the $k$ - th sensor at the FC. Denote by $\mathrm{RY}_{k}=\left[\mathrm{RY}_{k, 1}, \ldots \mathrm{RY} \mathrm{Y}_{k, N}\right]^{T}$ a vector of $N$ observations of $y$-location of the $k-t h$ sensor at the FC. Finally, the noisy observations of the field received by the FC are denoted as $Z_{k}, k=1, \ldots K$. All random variables in these sequences are mutually independent. The joint probability density function of these random variables will be:

$$
\begin{aligned}
f_{\mathbf{Z}, \mathbf{R X}, \mathbf{R Y}}(\mathbf{z}, \mathbf{r x}, \mathbf{r y})= & \prod_{k=1}^{K}\left[\prod_{n=1}^{N} f_{\mathrm{RX}}\left(\mathrm{rx}_{k, n}\right) f_{\mathrm{RY}}\left(\mathrm{ry}_{k, n}\right)\right] \times f_{Z}\left(z_{k}\right), \\
= & \prod_{k=1}^{K}\left[\prod_{n=1}^{N} \frac{1}{\sqrt{2 \pi\left(\xi^{2}+\eta^{2}\right)}} \exp \left(-\frac{\left(\mathrm{rx}_{k, n}-x_{k}\right)^{2}}{2\left(\tilde{\xi}^{2}+\eta^{2}\right)}\right)\right. \\
& \left.\frac{1}{\sqrt{2 \pi\left(\tilde{\xi}^{2}+\eta^{2}\right)}} \exp \left(-\frac{\left(\mathrm{ry} y_{k, n}-y_{k}\right)^{2}}{2\left(\tilde{\xi}^{2}+\eta^{2}\right)}\right)\right] \\
& \times \frac{1}{\sqrt{2 \pi\left(\sigma^{2}+\eta^{2}\right)}} \exp \left(-\frac{\left(z_{k}-G\left(x_{k}, y_{k}: \underline{\theta}\right)\right)^{2}}{2\left(\sigma^{2}+\eta^{2}\right)}\right) .
\end{aligned}
$$

The $\log$ of this pdf is:

$$
\begin{array}{r}
l(\mathbf{z}, \mathbf{r x}, \mathbf{r y})=\log \left\{\prod _ { k = 1 } ^ { K } \left[\frac{1}{\sqrt{2 \pi\left(\sigma^{2}+\eta^{2}\right)}} \exp \left(-\frac{\left(z_{k}-G\left(x_{k}, y_{k}: \underline{\theta}\right)\right)^{2}}{2\left(\sigma^{2}+\eta^{2}\right)}\right)\right.\right. \\
\left.\left.\times \prod_{n=1}^{N} \frac{1}{\sqrt{2 \pi\left(\xi^{2}+\eta^{2}\right)}} \exp \left(-\frac{\left(\mathrm{rx}_{k, n}-x_{k}\right)^{2}}{2\left(\tilde{\zeta}^{2}+\eta^{2}\right)}\right) \frac{1}{\sqrt{2 \pi\left(\tilde{\zeta}^{2}+\eta^{2}\right)}} \exp \left(-\frac{\left(\mathrm{ry} y_{k, n}-y_{k}\right)^{2}}{2\left(\xi^{2}+\eta^{2}\right)}\right)\right]\right\}
\end{array}
$$




$$
\begin{aligned}
l(\mathbf{z}, \mathbf{r x}, \mathbf{r y})= & -\frac{K}{2} \log \left(2 \pi\left(\sigma^{2}+\eta^{2}\right)\right)-\sum_{k=1}^{K} \frac{\left(z_{k}-G\left(x_{k}, y_{k}: \underline{\theta}\right)\right)^{2}}{2\left(\sigma^{2}+\eta^{2}\right)}-N K \log \left(2 \pi\left(\tilde{\zeta}^{2}+\eta^{2}\right)\right) \\
& -\sum_{k=1}^{K} \sum_{n=1}^{N}\left(\frac{\left(\mathrm{rx}_{k, n}-x_{k}\right)^{2}}{2\left(\tilde{\zeta}^{2}+\eta^{2}\right)}\right)-\sum_{k=1}^{K} \sum_{n=1}^{N}\left(\frac{\left(\mathrm{ry}_{k, n}-y_{k}\right)^{2}}{2\left(\tilde{\zeta}^{2}+\eta^{2}\right)}\right) .
\end{aligned}
$$

\subsubsection{Digital Modulation}

Due to a potential bandwidth constraint, the messages sent by sensors to the FC may need to be quantized. In this case, we have three types of data that need to be sent to the FC. The first two sets of data are the noisy measurements of the sensor location, which are provided by positioning technology system. Each estimate of sensor location is denoted by $\tilde{X}_{k}$ and $\tilde{Y}_{k}$. The other data are the measurements of the sensors, denoted as $R_{k}$. Therefore, quantization will be performed on these three different data sets separately. Let $Z_{k}$ be the received sensor measurement of the field at the receiver side, $\mathrm{RX}_{k}$ be the received noisy sensor position in $x$ dimension, $\mathrm{RY}_{k}$ be the received noisy sensor position in $y$ dimension and $W_{k, n}$ be AWGN. Below we first introduce the model to describe the received signals at the FC, then we will justify it. Denote by $T($.$) the transformation of an input$ data due to a uniform deterministic quantizer. Denote by $q$ the output of the quantizer. If the input to quantizer is a random variable, its output is a random variable too.

Consider a measurement $R_{k}$ of the $k$ - th sensor as an input to an M-level uniform deterministic quantizer. Its output $q_{k}$ is a discrete random variable with the following values and probability mass function:

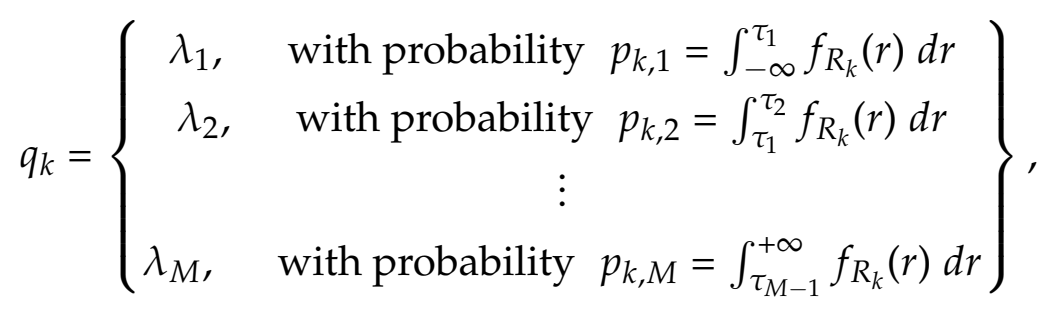

where $\lambda_{1}, \lambda_{2} \ldots, \lambda_{M}$ are known as reproduction points of the quantizer and $\left(\tau_{j}, \tau_{j+1}\right)$ are quantizer regions. For more details see [40].

In the following, quantized sensor measurements of the field will be denoted as $Q\left(R_{k}\right)$, where $k=1, \ldots, K$ and quantized estimated sensor position will be denoted as $Q\left(\tilde{X}_{k, n}\right)$ 
and $Q\left(\tilde{Y}_{k, n}\right)$, where $k=1, \ldots, K$ and $n=1, \ldots, N$.

We assume that the noise in the transmission channels is AWGN with variance $\eta^{2}$. Therefore, the received quantized observations are modeled as:

$$
\begin{aligned}
Z_{k} & =Q\left(R_{k}\right)+V_{k}, \\
\mathrm{RX}_{k, n} & =Q\left(\tilde{X}_{k, n}\right)+\mathrm{VX}_{k, n}, \\
\mathrm{RY}_{k, n} & =Q\left(\tilde{Y}_{k, n}\right)+V Y_{k, n},
\end{aligned}
$$

where the subindex $n$ indicates that more than one estimate of sensor positions is transmitted to the FC.

Since the transmission channels are parallel with independent noise samples and since the estimated sensor locations measurements and sensor measurements of the field are independent too, we can write a joint expression for the pdf of the data received by the FC as following:

$$
f_{Z, \mathrm{RX}, \mathrm{RY}}(\underline{z}, \underline{\mathrm{rX}}, \underline{\mathrm{ry}})=\prod_{k=1}^{K}\left[\prod_{n=1}^{N} f_{\mathrm{RX}}\left(\mathrm{rx}_{k, n}\right) f_{\mathrm{RY}}\left(\mathrm{ry}_{k, n}\right)\right] \times f_{\mathrm{Z}}\left(z_{k}\right) .
$$

Denote by $f_{Q_{R}}$ the probability density function of the quantized sensor measurements and $f_{Q_{X}}, f_{Q_{\tilde{Y}}}$ are the probability density functions of the quantized sensor locations measurements. Therefore, the marginal pdf of the quantized field measurements and quantized estimated sensor locations measurements are:

$$
\begin{aligned}
f_{Z}\left(z_{k}\right) & =f_{Q_{R}}\left(z_{k}\right) * f_{V_{k}}\left(z_{k}\right), \\
f_{\mathrm{RX}}\left(\mathrm{rx}_{k, n}\right) & =f_{Q_{\mathrm{RX}}}\left(\mathrm{rx}_{k, n}\right) * f_{\mathrm{VX}}\left(\mathrm{rx}_{k, n}\right), \\
f_{\mathrm{RY}}\left(\mathrm{ry}_{k, n}\right) & =f_{Q_{\mathrm{RY}}}\left(\mathrm{ry}_{k, n}\right) * f_{V Y_{k, n}}\left(\mathrm{ry}_{k, n}\right),
\end{aligned}
$$

where $*$ stands for convolution, $f_{Z_{k}}\left(z_{k}\right)$ is the probability density function of the quantized sensor measurement of the field, and $f_{\mathrm{RX}}\left(\mathrm{rx}_{k, n}\right), f_{\mathrm{RY}}\left(r, n y_{k, n}\right)$ are the probability density functions of the quantized sensor location measurements.

The pdf of $Q_{R_{k}}=q_{k}$ is given as:

$$
f_{q_{k}}(v)=\sum_{j=1}^{M} p_{k, j} \delta\left(v-\lambda_{j}\right),
$$


where $p_{k, j}$ is the probability for the output of the sensor $k$ to be mapped to the $j$-th reproduction point, and can be written as:

$$
p_{k, j}=\int_{\tau_{j}}^{\tau_{j+1}} \frac{1}{\sqrt{2 \pi \sigma^{2}}} \exp \left(-\frac{\left(t-G_{k}\right)^{2}}{2 \sigma^{2}}\right) d t,
$$

where $\tau_{j}$ and $\tau_{j+1}, j=1, \ldots, M$ are the boundaries of the $j$-th quantization region. The below equations describes the probability density function of the quantized field and sensor locations measurements:

$$
\begin{gathered}
f_{Q_{R_{k}}}\left(r_{k}\right)=\sum_{j=1}^{M} \int_{\tau_{j}}^{\tau_{j+1}} \frac{1}{\sqrt{2 \pi \sigma^{2}}} \exp \left(-\frac{\left(r-G\left(x_{k}, y_{k}: \underline{\theta}\right)\right)^{2}}{2 \sigma^{2}}\right) d r \delta\left(z_{k}-\lambda_{j}\right), \\
f_{Q_{\tilde{X}_{k, n}}}\left(r \mathrm{r}_{k, n}\right)=\sum_{j=1}^{M} \int_{\tau_{j}}^{\tau_{j+1}} \frac{1}{\sqrt{2 \pi \xi^{2}}} \exp \left(-\frac{\left(x-x_{k}\right)^{2}}{2 \tilde{\zeta}^{2}}\right) d x \delta\left(\mathrm{rx}_{k, n}-\lambda_{j}\right),
\end{gathered}
$$

and

$$
f_{Q_{\tilde{Y}_{k, n}}}\left(\mathrm{ry}_{k, n}\right)=\sum_{j=1}^{M} \int_{\tau_{j}}^{\tau_{j+1}} \frac{1}{\sqrt{2 \pi \tilde{\xi}^{2}}} \exp \left(-\frac{\left(y-y_{k}\right)^{2}}{2 \xi^{2}}\right) d y \delta\left(\mathrm{ry}_{k, n}-\lambda_{j}\right),
$$

by substituting $f_{Q_{R_{k}}}\left(r_{k}\right), f_{Q_{\tilde{X}_{k, n}}}\left(r x_{k, n}\right)$ and $f_{Q_{\tilde{Y}_{k, n}}}\left(r y_{k, n}\right)$ in eq.(3.17), eq.(3.18) and eq.(3.19), and performing convolution we obtain:

$$
\begin{aligned}
f_{Z_{k}}\left(z_{k}\right)= & \sum_{j=1}^{M}\left[Q\left(\frac{\left(\tau_{j}-G\left(x_{k}, y_{k}: \underline{\theta}\right)\right)}{\sigma}\right)-Q\left(\frac{\left(\tau_{j+1}-G\left(x_{k}, y_{k}: \underline{\theta}\right)\right)}{\sigma}\right)\right] \\
\times \frac{1}{\sqrt{2 \pi \eta^{2}}} \exp \left(-\frac{\left(z_{k}-\lambda_{j}\right)^{2}}{2 \eta^{2}}\right), & \\
f_{\mathrm{RX}_{k, n}}\left(\mathrm{rx}_{k, n}\right)= & \sum_{j=1}^{M}\left[Q\left(\frac{\left.\left(\tau_{j}-x_{k}\right)\right)}{\xi}\right)-Q\left(\frac{\left.\left(\tau_{j+1}-x_{k}\right)\right)}{\xi}\right)\right] \\
f_{\mathrm{RY}_{k, n}}\left(\mathrm{ry}_{k, n}\right)= & \sum_{j=1}^{M}\left[Q\left(\frac{\left.\left(\tau_{j}-y_{k}\right)\right)}{\xi}\right)-Q\left(\frac{\left.\left(\tau_{j+1}-y_{k}\right)\right)}{\xi}\right)\right] \\
& \times \frac{1}{\sqrt{2 \pi \eta^{2}}} \exp \left(-\frac{\left(\mathrm{rx}_{k, n}-\lambda_{j}\right)^{2}}{2 \eta^{2}}\right), \\
& \exp \left(-\frac{\left(\mathrm{ry}_{k, n}-\lambda_{j}\right)^{2}}{2 \eta^{2}}\right) .
\end{aligned}
$$


By substituting the equations (3.22), (3.23) and (3.24) in (3.16) we get the formula of the joint pdf at the FC:

$$
\begin{aligned}
f_{\mathrm{Z}, \mathrm{RX}, \mathrm{RY}}(\underline{z}, \underline{\mathrm{x}}, \underline{\mathrm{ry}})= & \prod_{k=1}^{K}\left(\sum_{j=1}^{M}\left[Q\left(\frac{\left(\tau_{j}-G\left(x_{k}, y_{k}: \underline{\theta}\right)\right)}{\sigma}\right)-Q\left(\frac{\left(\tau_{j+1}-G\left(x_{k}, y_{k}: \underline{\theta}\right)\right)}{\sigma}\right)\right]\right. \\
& \times \frac{1}{\sqrt{2 \pi \eta^{2}}} \exp \left(-\frac{\left(z_{k}-\lambda_{j}\right)^{2}}{2 \eta^{2}}\right) \prod_{n=1}^{N}\left(\sum _ { j = 1 } ^ { M } \left[Q\left(\frac{\left.\left(\tau_{j}-x_{k}\right)\right)}{\xi}\right)\right.\right. \\
& \left.\left.-Q\left(\frac{\left.\left(\tau_{j+1}-x_{k}\right)\right)}{\xi}\right)\right] \times \frac{1}{\sqrt{2 \pi \eta^{2}}} \exp \left(-\frac{\left(\mathrm{rx}_{k, n}-\lambda_{j}\right)^{2}}{2 \eta^{2}}\right)\right) \\
& \sum_{j=1}^{M}\left[Q\left(\frac{\left.\left(\tau_{j}-y_{k}\right)\right)}{\zeta}\right)-Q\left(\frac{\left.\left(\tau_{j+1}-y_{k}\right)\right)}{\xi}\right)\right] \\
& \left.\times \frac{1}{\sqrt{2 \pi \eta^{2}}} \exp \left(-\frac{\left(\mathrm{ry}_{k, n}-\lambda_{j}\right)^{2}}{2 \eta^{2}}\right)\right) .
\end{aligned}
$$

The log-likelihood of the likelihood in (3.25) can be written as:

$$
\begin{aligned}
l_{\mathrm{Z}, \mathrm{RX}, \mathrm{RY}}(\underline{z}, \underline{\mathrm{rx}}, \underline{\mathrm{ry}})= & \sum_{k=1}^{K}\left(\log \sum_{j=1}^{M}\left[Q\left(\frac{\left(\tau_{j}-G\left(x_{k}, y_{k}: \underline{\theta}\right)\right)}{\sigma}\right)-Q\left(\frac{\left.\left(\tau_{j+1}-G\left(x_{k}, y_{k}: \underline{\theta}\right)\right)\right)}{\sigma}\right)\right]\right. \\
& \times \frac{1}{\sqrt{2 \pi \eta^{2}}} \exp \left(-\frac{\left(z_{k}-\lambda_{j}\right)^{2}}{2 \eta^{2}}\right)+\sum_{n=1}^{N} \log \left(\sum _ { j = 1 } ^ { M } \left[Q\left(\frac{\left.\left(\tau_{j}-x_{k}\right)\right)}{\xi}\right)\right.\right. \\
& \left.\left.-Q\left(\frac{\left.\left(\tau_{j+1}-x_{k}\right)\right)}{\xi}\right)\right] \times \frac{1}{\sqrt{2 \pi \eta^{2}}} \exp \left(-\frac{\left(\mathrm{rx}_{k, n}-\lambda_{j}\right)^{2}}{2 \eta^{2}}\right)\right) \\
& +\sum_{n=1}^{N} \log \left(\sum_{j=1}^{M}\left[Q\left(\frac{\left.\left(\tau_{j}-y_{k}\right)\right)}{\xi}\right)-Q\left(\frac{\left.\left(\tau_{j+1}-y_{k}\right)\right)}{\xi}\right)\right]\right. \\
& \left.\times \frac{1}{\sqrt{2 \pi \eta^{2}}} \exp \left(-\frac{\left(\mathrm{ry}_{k, n}-\lambda_{j}\right)^{2}}{2 \eta^{2}}\right)\right) .
\end{aligned}
$$

\subsubsection{Justification of the Model for Transmission Channels}

To justify the model describing the data at the FC, we consider the signal processing for communications in details. For simplicity we consider On-Off Keying (OOK) modulation and coherent demodulation of the received signal. However, a similar model can be derived for other linear modulation schemes. 
To apply OOK modulation, the data have to be presented in a binary form. Each reproduction point $\lambda_{j}, j=1, \ldots, M$ of the quantizer is presented as one of integers $1, \ldots, M$ and encoded using $\log _{2}(M)$ bits.

Since $Q\left(R_{k}\right)$ is a random variable and $Q\left(R_{k}\right)=\lambda_{j}, j=1, \ldots, M$ occurs with probability $p_{k, j}$, the prior probability on the encoded $Q_{R_{k}}$ is also equal to $p_{k, j}$. Denote by $\underline{B}_{k}$ a random binary codeword for $Q\left(R_{k}\right)$. The codeword can take values $\underline{b}_{k, j}$ with probability $p_{k, j}$, where $j=1, \ldots, M$. The codeword $\underline{B}_{k}$ is modulated using OOK modulation to a known frequency $f_{c}$.

The modulated vector signal

$$
\underline{B}_{k}(t)=\underline{B}_{k} \cos \left(2 \pi f_{c} t\right)
$$

is transmitted over a AWGN channel, bit by bit, to the FC. Thus, the received vector signal

$$
\underline{R B}_{k}(t)=\underline{B}_{k}(t)+\underline{V}_{k}(t),
$$

where $\underline{V}_{k}(t)$ is a vector random signal composed of i.i.d white Gaussian noise processes with zero mean and power spectral density $\frac{N_{0}}{2}$. Note that each bit is transmitted and received over an interval of length $\mathrm{T}$. The intervals do not overlap.

To extract a vector representation in a signal space, we assume that $\underline{R B}{ }_{k}(t)$ is passed through a correlator or a matched filter. Then the received signal is

$$
\begin{aligned}
\underline{R B}_{k}(t) & =\frac{2}{T} \int_{0}^{T} \underline{R B}_{k}(t) \cos \left(2 \pi f_{c} t\right) d t . \\
& =\frac{2}{T}\left[\int_{0}^{T} \underline{B}_{k} \cos ^{2}\left(2 \pi f_{c} t\right) d t+\int_{0}^{T} \underline{V}_{k}(t) \cos \left(2 \pi f_{c} t\right) d t\right] .
\end{aligned}
$$

Denote by $\underline{V}_{k}$ a vector of i.i.d Gaussian random variables with mean zero and variance 


$$
\begin{aligned}
\sigma_{V_{k}}^{2} & =E\left[\int_{0}^{T} \int_{0}^{T} V_{k}(t) V_{k}(u) \cos \left(2 \pi f_{c} t\right) \cos \left(2 \pi f_{c} u\right) d t d u\right] \\
& =\int_{0}^{T} \int_{0}^{T} E\left[V_{k}(t) V_{k}(u)\right] \cos \left(2 \pi f_{c} t\right) \cos \left(2 \pi f_{c} u\right) d t d u \\
& =\frac{N_{o}}{2} \int_{0}^{T} \int_{0}^{T} \delta(t-u) \cos \left(2 \pi f_{c} t\right) \cos \left(2 \pi f_{c} u\right) d t d u \\
& =\frac{N_{o}}{2} \int_{0}^{T} \cos ^{2}\left(2 \pi f_{c} t\right) d t \\
& =\frac{N_{o}}{2}\left[\frac{T}{2}+\frac{1}{2} \int_{0}^{T} \cos \left(4 \pi f_{c} t\right) d t\right] .
\end{aligned}
$$

If $T$ is selected such that $T=m \frac{1}{f_{c}}, \mathrm{~m}$ is a positive integer, then

$$
\sigma_{V_{k}}^{2}=\frac{N_{o}}{T}
$$

and

$$
\underline{R B}_{k}=\underline{B}_{k}+\underline{V}_{k}
$$

This justifies the use of Gaussian models for the noise in transmission channels of the WSN. The block diagram detailing the signal processing for the transmission of the quantized field observations to the FC is shown in Fig. 3.2.

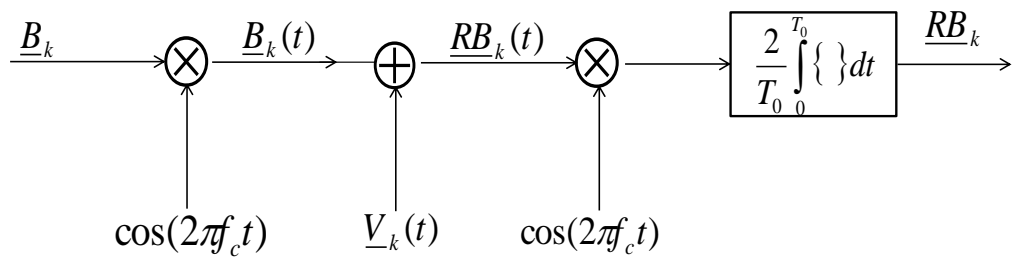

Figure 3.2: Signal processing for digital modulation and demodulation. 


\subsection{Proposed Solution}

In this section we will discuss the used algorithms to solve the problem stated in the previous section. The used estimation method is MLE method [41, 42]. Since the likelihood function is the joint probability of the observed data, MLE will look for the value of parameter $\underline{\theta}$ that maximizes the likelihood function. After formulating the MLE solution, an iterative method to solve a set of nonlinear equations is proposed using a bisection algorithm.

\subsubsection{Maximum Likelihood Solution for Analog Case}

Maximum Likelihood is an estimation approach that maximizes the likelihood function (joint probability density function) of the observed data in order to estimate the unknown parameters of the received data [41,42]. The MLE solution is using the joint pdf obtained in section 3.1.1 to estimate the unknown parameters that maximize the $\log$ likelihood in eq.(3.12). The estimated parameters $\underline{\hat{\theta}}_{M L}$ is the solution to the following optimization problem:

$$
\underline{\hat{\theta}}_{M L}=\arg \max _{\underline{\theta} \in \Theta} l(\mathbf{Z}, \mathbf{R X}, \mathbf{R Y}: \underline{\theta}),
$$

where $\Theta$ is a set of admissible solutions. The necessary condition solve eq.(3.33) is as follows:

$$
\left.\nabla_{l_{\underline{\theta}}}(\mathbf{Z}, \mathbf{R X}, \mathbf{R Y})\right|_{\underline{\hat{\theta}}}=0
$$

The unknown parameters in our problem $\underline{\theta}$ include the unknown sensors positions $\left[\left(x_{1}, y_{1}\right) \ldots\left(x_{k}, y_{k}\right)\right]^{T}, k=1,2, \ldots K$ and the unknown location of the point source $\left(x_{c}, y_{c}\right)$. The total number of the unknown parameters is $(2 K+2)$. For the estimation problem to be well posed, we need the same or larger number of data points. Therefore, we assume that we have $N$ estimates of each sensor position. Taking the derivatives of the log-likelihood function in eq.(3.12) with respect to the true sensor locations and applying the necessary 
conditions yields:

$$
\begin{aligned}
& \frac{\partial l(\mathbf{Z}, \mathbf{R X}, \mathbf{R Y})}{\partial x_{k}}=-\sum_{k=1}^{K} \frac{\left(z_{k}-G\left(x_{k}, y_{k}: \underline{\theta}\right)\right)}{\left(\sigma^{2}+\eta^{2}\right)}\left(\frac{-\partial G\left(x_{k}, y_{k}: \underline{\theta}\right)}{\partial x_{k}}\right) \\
&-\sum_{k=1}^{K} \sum_{n=1}^{N}\left(\frac{-\left(r x_{k, n}-x_{k}\right)}{\xi^{2}}\right)=0, \\
& \frac{\partial G\left(x_{k}, y_{k}: \underline{\theta}\right)}{\partial x_{k}}=-h \frac{\left(x_{k}-x_{c}\right)}{\sigma_{g}^{2}} \exp \left(\frac{-\left(x_{k}-x_{c}\right)^{2}-\left(y_{k}-y_{c}\right)^{2}}{2 \sigma_{g}^{2}}\right),
\end{aligned}
$$

where $\sigma_{g}^{2}$ the spread of the field.

and

$$
\begin{aligned}
\frac{\partial l(\mathbf{Z}, \mathbf{R X}, \mathbf{R Y})}{\partial y_{k}}= & -\sum_{k=1}^{K} \frac{\left(z_{k}-G\left(x_{k}, y_{k}: \underline{\theta}\right)\right)}{\left(\sigma^{2}+\eta^{2}\right)}\left(\frac{-\partial G\left(x_{k}, y_{k}: \underline{\theta}\right)}{\partial y_{k}}\right) \\
& -\sum_{k=1}^{K} \sum_{n=1}^{N}\left(\frac{-\left(\mathrm{ry}_{k, n}-y_{k}\right)}{\tilde{\xi}^{2}}\right)=0,
\end{aligned}
$$

where

$$
\frac{\partial G\left(x_{k}, y_{k}: \underline{\theta}\right)}{\partial y_{k}}=-h \frac{\left(y_{k}-y_{c}\right)}{\sigma_{g}^{2}} \exp \left(\frac{-\left(x_{k}-x_{c}\right)^{2}-\left(y_{k}-y_{c}\right)^{2}}{2 \sigma_{g}^{2}}\right) .
$$

The derivatives of the $\log$-likelihood function with respect to the field parameters are given as:

$$
\frac{\partial l(\mathbf{Z}, \mathbf{R X}, \mathbf{R Y})}{\partial x_{c}}=-\sum_{k=1}^{K} \frac{\left(z_{k}-G\left(x_{k}, y_{k}: \underline{\theta}\right)\right)}{\left(\sigma^{2}+\eta^{2}\right)}\left(\frac{-\partial G\left(x_{k}, y_{k}: \underline{\theta}\right)}{\partial x_{c}}\right)=0
$$

where

$$
\frac{\partial G\left(x_{k}, y_{k}: \underline{\theta}\right)}{\partial x_{c}}=h \frac{\left(x_{k}-x_{c}\right)}{\sigma_{g}^{2}} \exp \left(\frac{-\left(x_{k}-x_{c}\right)^{2}-\left(y_{k}-y_{c}\right)^{2}}{2 \sigma_{g}^{2}}\right),
$$

and

$$
\frac{\partial l(\mathbf{Z}, \mathbf{R X}, \mathbf{R Y})}{\partial y_{c}}=-\sum_{k=1}^{K} \frac{\left(z_{k}-G\left(x_{k}, y_{k}: \underline{\theta}\right)\right)}{\left(\sigma^{2}+\eta^{2}\right)}\left(\frac{-\partial G\left(x_{k}, y_{k}: \underline{\theta}\right)}{\partial y_{c}}\right)=0
$$

where

$$
\frac{\partial G\left(x_{k}, y_{k}: \underline{\theta}\right)}{\partial y_{c}}=h \frac{\left(y_{k}-y_{c}\right)}{\sigma_{g}^{2}} \exp \left(\frac{-\left(x_{k}-x_{c}\right)^{2}-\left(y_{k}-y_{c}\right)^{2}}{2 \sigma_{g}^{2}}\right) .
$$




\subsubsection{Maximum Likelihood Solution for Digital Case}

The necessary conditions to find the MLE for digital case are as follows:

$$
\left.\nabla_{l_{\underline{\theta}}}(\mathbf{Z}, \mathbf{R X}, \mathbf{R} \mathbf{Y})\right|_{\underline{\hat{\theta}}}=0 \text {. }
$$

The derivatives of the $\log$-likelihood function with respect to the sensor locations are as follows:

$$
\frac{\partial l(\mathbf{Z}, \mathbf{R X}, \mathbf{R Y})}{\partial x_{k}}=\sum_{k=1}^{K}\left[\frac{\frac{\partial f_{Z}\left(z_{k}\right)}{\partial x_{k}}}{f_{Z}\left(z_{k}\right)}+\sum_{n=1}^{N} \frac{\frac{\partial f_{\mathrm{RX}}\left(\mathrm{r}_{k, n}\right)}{\partial x_{k}}}{f_{\mathrm{RX}}\left(\mathrm{rx}_{k, n}\right)}\right]
$$

where

$$
\begin{gathered}
\frac{\partial f_{Z_{k}}\left(z_{k}\right)}{\partial x_{k}}=\sum_{j=1}^{M}\left(\exp \left(\frac{-\left(\tau_{j}-G\left(x_{k}, y_{k}: \underline{\theta}\right)\right)}{2 \sigma^{2}}\right)-\exp \left(\frac{-\left(\tau_{j+1}-G\left(x_{k}, y_{k}: \underline{\theta}\right)\right)}{2 \sigma^{2}}\right)\right) \\
\quad \times \frac{1}{\sqrt{2 \pi \sigma^{2}}}\left(\frac{\partial G\left(x_{k}, y_{k}: \underline{\theta}\right)}{\partial x_{k}}\right) \frac{1}{\sqrt{2 \pi \eta^{2}}} \exp \left(-\frac{\left(z_{k}-\lambda_{j}\right)^{2}}{2 \eta^{2}}\right), \\
\frac{\partial G\left(x_{k}, y_{k}: \underline{\theta}\right)}{\partial x_{k}}=-h \frac{\left(x_{k}-x_{c}\right)}{\sigma_{g}^{2}} \exp \left(-\frac{\left(x_{k}-x_{c}\right)^{2}-\left(y_{k}-y_{c}\right)^{2}}{2 \sigma_{g}^{2}}\right),
\end{gathered}
$$

and

$$
\begin{aligned}
\frac{\partial f_{\mathrm{RX} \mathrm{k}_{\mathrm{n}}}\left(\mathrm{rx}_{k, n}\right)}{\partial x_{k}}= & \sum_{j=1}^{M}\left(\exp \left(\frac{-\left(\tau_{j}-x_{k}\right)}{2 \xi^{2}}\right)-\exp \left(\frac{-\left(\tau_{j+1}-x_{k}\right)}{2 \xi^{2}}\right)\right) \\
& \times \frac{1}{\sqrt{2 \pi \eta^{2}}} \exp \left(-\frac{\left(\mathrm{rx}_{k, n}-\lambda_{j}\right)^{2}}{2 \eta^{2}}\right),
\end{aligned}
$$

and

$$
\frac{\partial l(\mathbf{Z}, \mathbf{R X}, \mathbf{R Y})}{\partial y_{k}}=\sum_{k=1}^{K}\left[\frac{\frac{\partial f_{Z}\left(z_{k}\right)}{\partial y_{k}}}{f_{Z}\left(z_{k}\right)}+\sum_{n=1}^{N} \frac{\frac{\partial f_{\mathrm{RY}}\left(r y_{k, n}\right)}{\partial y_{k}}}{f_{\mathrm{RY}}\left(r \mathrm{r}_{k, n}\right)}\right]
$$

where

$$
\begin{aligned}
\frac{\partial f_{Z_{k}}\left(z_{k}\right)}{\partial y_{k}}= & \sum_{j=1}^{M}\left(\exp \left(\frac{-\left(\tau_{j}-G\left(x_{k}, y_{k}: \underline{\theta}\right)\right)}{2 \sigma^{2}}\right)-\exp \left(\frac{-\left(\tau_{j+1}-G\left(x_{k}, y_{k}: \underline{\theta}\right)\right)}{2 \sigma^{2}}\right)\right) \\
& \times \frac{1}{\sqrt{2 \pi \sigma^{2}}}\left(\frac{\partial G\left(x_{k}, y_{k}: \underline{\theta}\right)}{\partial y_{k}}\right) \frac{1}{\sqrt{2 \pi \eta^{2}}} \exp \left(-\frac{\left(z_{k}-\lambda_{j}\right)^{2}}{2 \eta^{2}}\right),
\end{aligned}
$$




$$
\frac{\partial G\left(x_{k}, y_{k}: \underline{\theta}\right)}{\partial y_{k}}=-h \frac{\left(y_{k}-y_{c}\right)}{\sigma_{g}^{2}} \exp \left(\frac{-\left(x_{k}-x_{c}\right)^{2}-\left(y_{k}-y_{c}\right)^{2}}{2 \sigma_{g}^{2}}\right)
$$

and

$$
\begin{aligned}
\frac{\partial f_{\mathrm{RY}_{k, n}}\left(\mathrm{ry}_{k, n}\right)}{\partial y_{k}}= & \sum_{j=1}^{M}\left(\exp \left(\frac{-\left(\tau_{j}-y_{k}\right)}{2 \xi^{2}}\right)-\exp \left(\frac{-\left(\tau_{j+1}-y_{k}\right)}{2 \xi^{2}}\right)\right) \\
& \times \frac{1}{\sqrt{2 \pi \eta^{2}}} \exp \left(-\frac{\left(\mathrm{ry}_{k, n}-\lambda_{j}\right)^{2}}{2 \eta^{2}}\right) .
\end{aligned}
$$

The derivatives of the $\log$-likelihood function with respect to the target location are given as:

$$
\frac{\partial l(\mathbf{Z}, \mathbf{R X}, \mathbf{R Y})}{\partial x_{c}}=\sum_{k=1}^{K} \frac{\frac{\partial f_{Z}\left(z_{k}\right)}{\partial x_{c}}}{f_{Z}\left(z_{k}\right)}
$$

where

$$
\begin{gathered}
\frac{\partial f_{Z_{k}}\left(z_{k}\right)}{\partial x_{c}}=\sum_{j=1}^{M}\left(\exp \left(\frac{-\left(\tau_{j}-G\left(x_{k}, y_{k}: \underline{\theta}\right)\right)}{2 \sigma^{2}}\right)-\exp \left(\frac{-\left(\tau_{j+1}-G\left(x_{k}, y_{k}: \underline{\theta}\right)\right)}{2 \sigma^{2}}\right)\right) \\
\quad \times \frac{1}{\sqrt{2 \pi \sigma^{2}}}\left(\frac{\partial G\left(x_{k}, y_{k}: \underline{\theta}\right)}{\partial x_{c}}\right) \frac{1}{\sqrt{2 \pi \eta^{2}}} \exp \left(-\frac{\left(z_{k}-\lambda_{j}\right)^{2}}{2 \eta^{2}}\right), \\
\frac{\partial G\left(x_{k}, y_{k}: \underline{\theta}\right)}{\partial x_{c}}=h \frac{\left(x_{k}-x_{c}\right)}{\sigma_{g}^{2}} \exp \left(\frac{-\left(x_{k}-x_{c}\right)^{2}-\left(y_{k}-y_{c}\right)^{2}}{2 \sigma_{g}^{2}}\right),
\end{gathered}
$$

and

$$
\frac{\partial l(\mathbf{Z}, \mathbf{R X}, \mathbf{R Y})}{\partial y_{c}}=\sum_{k=1}^{K} \frac{\frac{\partial f_{Z}\left(z_{k}\right)}{\partial y_{c}}}{f_{Z}\left(z_{k}\right)}
$$

where

$$
\begin{aligned}
\frac{\partial f_{Z_{k}}\left(z_{k}\right)}{\partial y_{c}}= & \sum_{j=1}^{M}\left(\exp \left(\frac{-\left(\tau_{j}-G\left(x_{k}, y_{k}: \underline{\theta}\right)\right)}{2 \sigma^{2}}\right)-\exp \left(\frac{-\left(\tau_{j+1}-G\left(x_{k}, y_{k}: \underline{\theta}\right)\right)}{2 \sigma^{2}}\right)\right) \\
& \times \frac{1}{\sqrt{2 \pi \sigma^{2}}}\left(\frac{\partial G\left(x_{k}, y_{k}: \underline{\theta}\right)}{\partial y_{c}}\right) \frac{1}{\sqrt{2 \pi \eta^{2}}} \exp \left(-\frac{\left(z_{k}-\lambda_{j}\right)^{2}}{2 \eta^{2}}\right),
\end{aligned}
$$

and

$$
\frac{\partial G\left(x_{k}, y_{c}: \underline{\theta}\right)}{\partial y_{c}}=h \frac{\left(y_{k}-y_{c}\right)}{\sigma_{g}^{2}} \exp \left(\frac{-\left(x_{k}-x_{c}\right)^{2}-\left(y_{k}-y_{c}\right)^{2}}{2 \sigma_{g}^{2}}\right) .
$$




\subsubsection{Iterative Solution}

The algorithm used in the thesis to find the roots of the $\log$-likelihood function to determine the position $\left(x_{k}, y_{k}\right)$ of the sensors as well as the target location $\left(x_{c}, y_{c}\right)$ is the bisection algorithm [43, 44, 45]. Bisection algorithm is an iterative method to solve for roots of nonlinear equations. Suppose we need to find roots of a non-linear function $f(x)$. This algorithm is performed by assuming two initial values, for instance $a$ and $b$, for the argument of a function $f(x)$. The function $f(x)$ evaluated at $a$ and $b$. Takes values of the opposite sign this ensures that the root of the function is somewhere between these two values. To iteratively approach the root of a nonlinear equation involving $f(x)$, a new value of the argument $x$ is introduced. The new value $c$ is set to a mid-value between $a$ and $b, \frac{a+b}{2}$. After substituting the value of $c$ in the function $f(x)$, we check the sign of $f(c)$. If $f(c)$ is positive, then $c$ will replace $a$, otherwise it will replace $b$. This procedure will continue till the method reaches the root of the function $f(x)$. The main advantage of the bisection algorithm is the robustness of the algorithm and the accuracy of results. The main contribution we made in this part of the thesis is in the improvement of the accuracy of the bisection algorithm by incorporating new constraints. The new constraints will prevent the system from picking initial values that do not meet the requirement. The flow chart explains how the bisection algorithm works with the new constraints. 


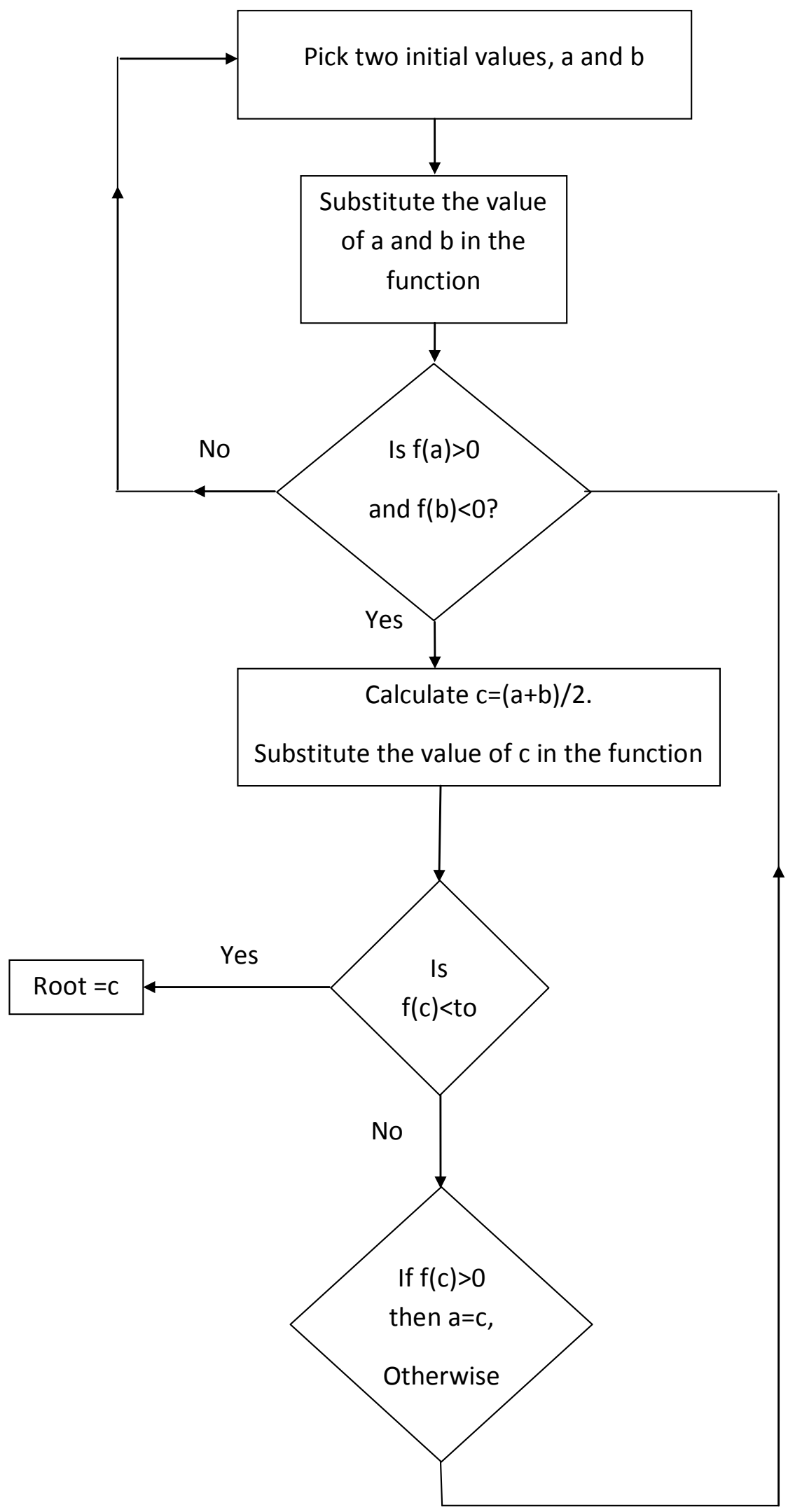

Figure 3.3: Flow chart of the bisection algorithm. 


\section{Chapter 4}

\section{Numerical Results}

In this chapter we describe our experimental setup and analyze the performance of the proposed algorithm through Monte Carlo simulations. In all experiments below the goal is to estimate the position of an object of interest. In this chapter, the object is characterized by a parametric physical field modeled as a Gaussian bell. However, the framework for distributed parameter estimation developed in the previous chapters can be applied to any parametric field. The estimation performance will be evaluated using a square error measure defined as:

$$
S E=\left(x_{c}-\hat{x}_{c}\right)^{2}+\left(y_{c}-\hat{y}_{c}\right)^{2} \text {. }
$$

In our experiments a distributed network of $\mathrm{K}$ sensors are deployed over an area $\mathrm{A}$ of size $10 \times 10$. A field generated by an object is modeled as a Gaussian bell :

$$
G(x, y)=\Lambda \exp \left(-\frac{\left(x-x_{c}\right)^{2}+\left(y-y_{c}\right)^{2}}{2 \sigma_{g}^{2}}\right),
$$

where $\Lambda$ is the field strength and its value in this simulation is assumed to be 100 , and the value of $\sigma_{g}^{2}$ is fixed at 5 .

Each sensor takes a measurement of the field and reports its measurement to the FC via a noisy wireless channel. The channels are assumed to be AWGN channels. The unknown parameters in this problem are the target location $\left(x_{c}, y_{c}\right)$ and the true sensor locations $\left(x_{k}, y_{k}\right), k=1, \ldots, K$. 
Many results of this chapter are described using a box plot. Box plot is a graphical method to describe a certain range of data. A box plot has the following features:

- The top and bottom of the box are the 25th and 75th percentiles of the observations.

- The line in the middle of the box is the median of the observations.

- The lines above and under the box are the maximum and the minimum of the observations.

- The observations that are above the maximum and marked by a red plus signs ++ are called outliers.

In this chapter we will present the simulation results for analog and digital case.

\subsection{Auxiliary Performance Measures}

In this section we will define certain performance measures such as the probability of outliers and signal to noise ratio for the analog and digital cases assumed in our simulations.

\subsubsection{Probability of Outliers}

Outliers are defined as the values of SE that lie above the maximum value in a box plot. The Probability of outliers is defined here as the probability that the square error is above a certain threshold $\tau>0$ :

$$
P_{\text {outliers }}(\tau)=P[S E>\tau] .
$$

\subsubsection{Signal to Noise Ratio for Analog Channel}

When sensors take measurements of the field, these measurements are mixed with the noise in channel. All numerical illustrations presented in this chapter rely on the assumption of AWGN in the observation and transmission channels. However, the basic 
Gaussian models can be easily generalized to include fading and shadowing effects as well as the effect of interference.

In order to characterize the strength of each signal and compare it to the strength of the noise, we define signal-to-noise ratio in observation channel, $\left(S N R_{o}\right)$ :

$$
S N R_{o}=\frac{\iint_{A} G(x, y: \theta)^{2} d x d y}{A \sigma^{2}} .
$$

For majority of simulations, $S N R_{o}$ is set to $20 \mathrm{~dB}$. Therefore from eq.(4.4) the noise variance in the observation channel is set to $\sigma^{2}=14.9217$.

In addition to that, after the sensors collect the information about the field they need to send this information to the FC over AWGN channels with variance $\eta^{2}$. To characterize distortions encountered by sensor measurements during their transmission, we define SNR of the transmission channel, $S N R_{c}$ :

$$
S N R_{c}=\frac{\iint_{A} E\left[R^{2}(x, y)\right] d x d y}{A \eta^{2}} .
$$

In many experiments we set $S N R_{c}$ to $20 \mathrm{~dB}$. Therefore, from eq.(4.5) the noise variance in the communication channel is $\eta^{2}=15.0710$.

\subsubsection{Signal to Noise Ratio for Digital Channel}

Since the measurements taken by sensors in the case of analog and digital modulations are the same, the $S N R_{o}$ is also the same:

$$
S N R_{o}=\frac{\iint_{A} G(x, y: \theta)^{2} d x d y}{A \sigma^{2}} .
$$

On the other hand, Signal to Noise Ratio in the communication channel $S N R_{c}$ under the digital modulation will be different, because of the quantization applied to measurements prior to their transmission. Therefore, $S N R_{c}$ in the case of digital modulation is defined as:

$$
S N R_{c}=\frac{\iint_{A} E\left[q^{2}(R(x, y))\right] d x d y}{A \eta^{2}} .
$$


It is set to $20 \mathrm{~dB}$ in simulations. Therefore, from eq.(4.7) the variance of the noise in a communication channel is $\eta^{2}=0.1271$.

\subsection{Numerical Results for Analog Case}

In this section we evaluate the performance of the proposed algorithm when the system is sending raw data (non-quantized data) to the FC. We will investigate how different parameters of the WSN affect the performance of the distributed estimator.

\subsubsection{The Effect of Varying the Number of Sensors}

In this experiment, we evaluate how the sensor density will affect the performance of target location estimation. All remaining parameters in this experiment will be fixed. The number of sensors is varied as 10, 20, 30, 50, 70, 90. The number of estimated sensor location measurements per sensor is fixed at $N=5$, the variance of the estimated measurements of sensor positions is set to $\xi^{2}=0.4$, and the variances of the noise in observation and transmission channels are set to $\sigma^{2}=14.9217$ and $\eta^{2}=15.0710$. After performing this experiment, we noticed that with increasing the number of sensors over a fixed area the value of the square error is decreasing. From Fig. 4.1 we also note that varying the number of sensors from $K=10$ to $K=20$ has a significant effect on the performance of the estimator, but this effect becomes less significant when the number of sensors increases to $K>50$.

\subsubsection{Varying the Strength of the Field $\Lambda$}

In this experiment we show how the strength of the field can influence the performance of the distributed estimator. The number of sensors is fixed at $K=50$ and the number of estimated sensor location measurements per sensor is fixed at $N=5$. The field strength $\Lambda$ is varied in the range $10,30,50,70,90,100$. Since the strength of the field has a major effect on the signal to noise ratio, it is expected that increasing the strength of the field will improve the performance of the estimator, and it is confirmed by the con- 


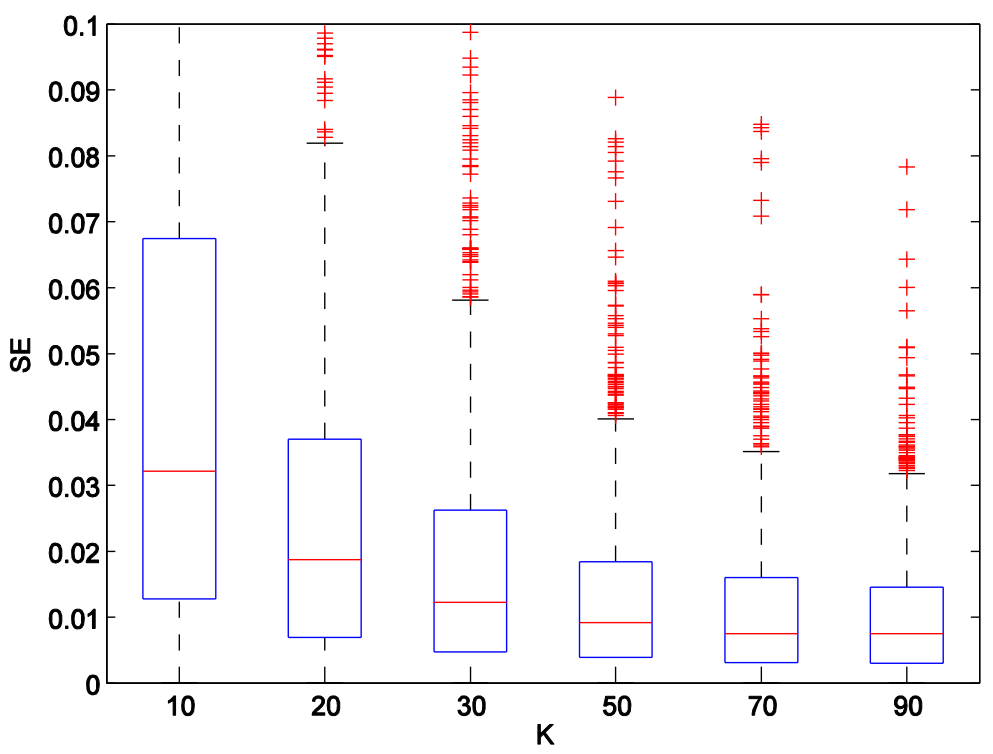

Figure 4.1: Dependence of the simulated SE on the number of sensors.

ducted experiment. From the box plot in Fig. 4.2 we also observe that when the strength of the field is above 70, $\Lambda>70$, varying the strength of the field has a minor effect on the performance of the estimator.

\subsubsection{The Probability of Outliers with Varying the Number of Sensors}

In this section we are varying the number of sensors and evaluating the probability of outliers. The experiment is conducted for $K=10,30,70$. The value of the threshold is varied from 0 to 1 with unit step of 0.1 . As expected, from Fig. 4.3 the probability of outliers decreases when the threshold value $\tau$ increases. We also notice that, with the number of sensors increasing the probability of outliers decreasing.

\subsubsection{Comparison with the Baseline Case when Sensor Positions are Known}

To evaluate performance degradation due to the uncertainty in sensor locations, we compare our results with a clairvoyant case, when position of sensors are known to the 


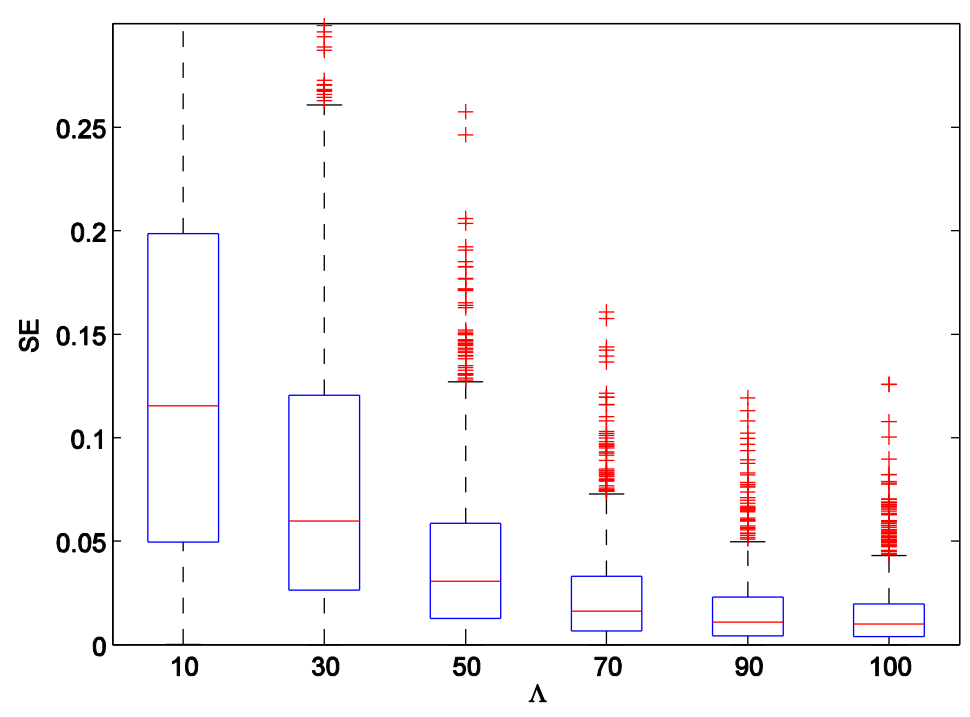

Figure 4.2: Dependence of the simulated SE on the strength of the field.

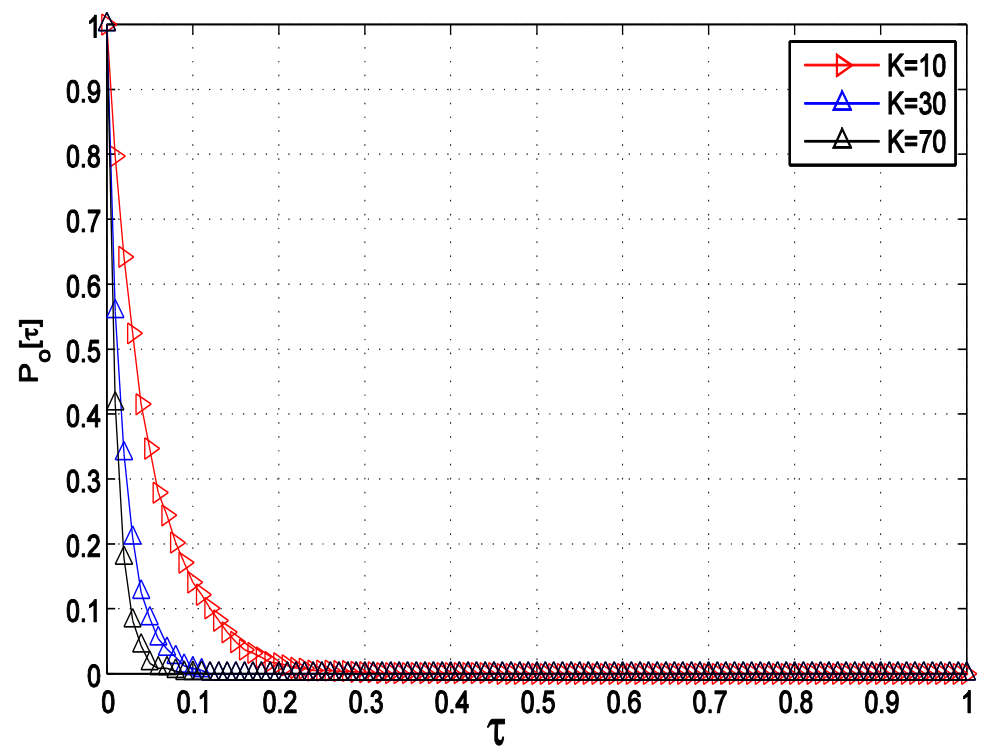

Figure 4.3: Dependence of the probability of outliers on the threshold $\tau$ parametrized by three different values of $\mathrm{K}$.

FC. Fig. 4.4 shows the results of the comparison. The number of sensors is fixed at $K=50$. The below box plot shows the clairvoyant case where there is certainty in sensor locations and for the case when the FC uses $N=5$ number of estimated measurements of sensor 
location per sensor where there is uncertainty in sensor locations.

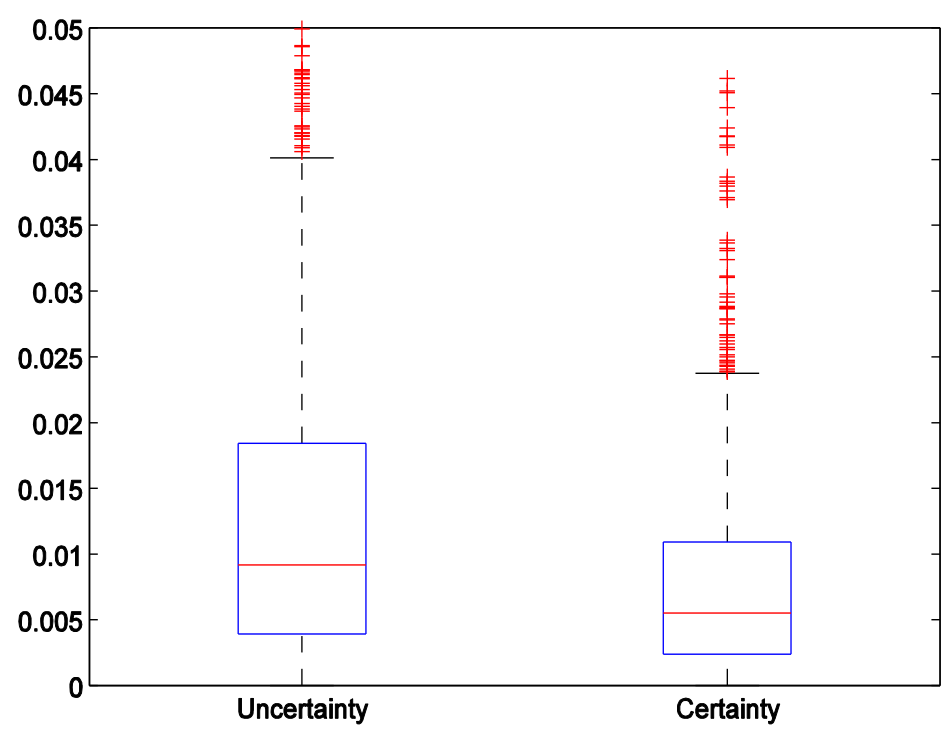

Figure 4.4: The performance comparison between estimators with certainty and uncertainty in sensor locations. The network is composed of $K=50$ sensors.

\subsubsection{Varying Signal to Noise Ratio}

In this part of the numerical results we vary the value of the signal to noise ratio and evaluate its effect on the performance of the proposed estimation algorithm. In this case the number of sensors is fixed at $K=50$ and the number of estimated sensor measurement is set to $N=5$. In order to perform this simulation the observation signal to noise ratio $S N R_{o}$ and the transmission signal to noise ratio $S N R_{c}$ are set to different values $S N R_{0}=S N R_{c}=10,15,20$. From the generated box plot we notice that the square error of target estimation is decreasing with increasing signal to noise ratio, as expected. 


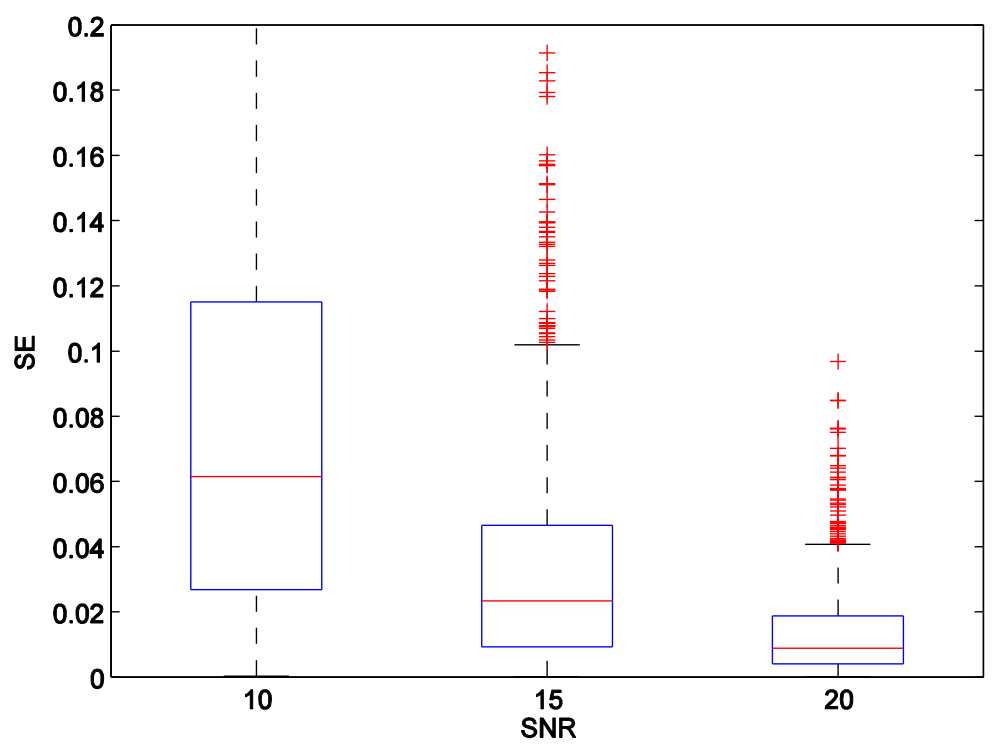

Figure 4.5: Dependence of the simulated SE on the signal to noise ratio.

We also evaluate the probability of outliers for the same three values of signal to noise ratio. The results of this simulation are shown in Fig. 4.6.

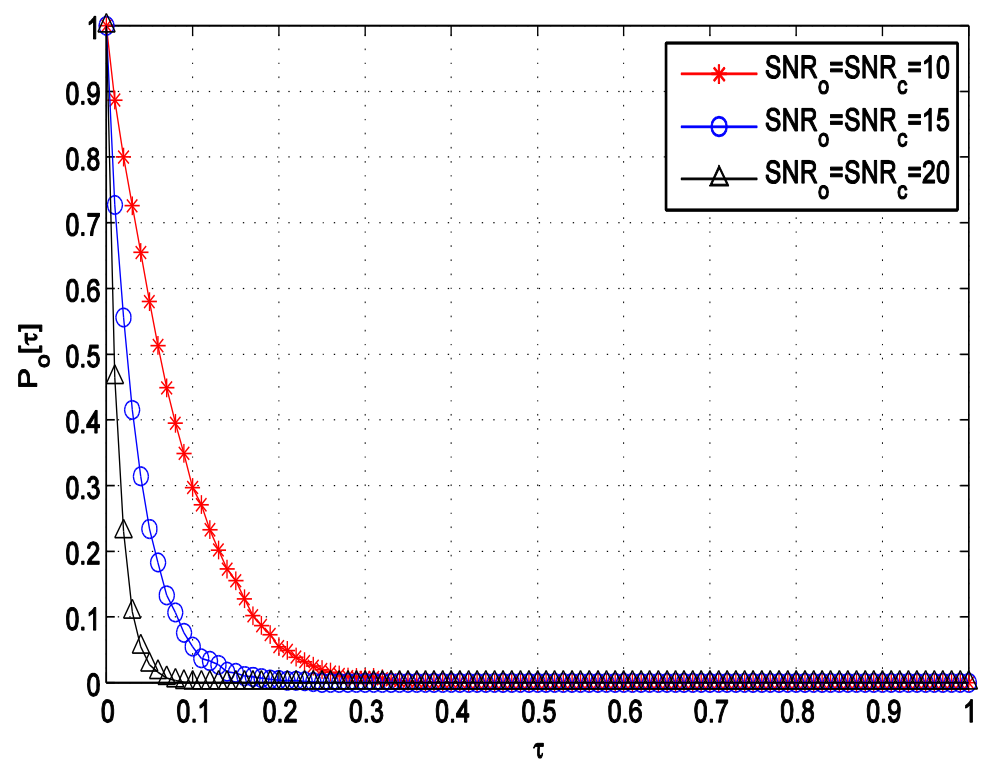

Figure 4.6: Dependence of the probability of outliers on the value of $\tau$. The plots are parametrized by three different values of SNR. 
As well, in Fig. 4.7 we also observe the effect of varying SNR on the performance of estimating sensor locations.

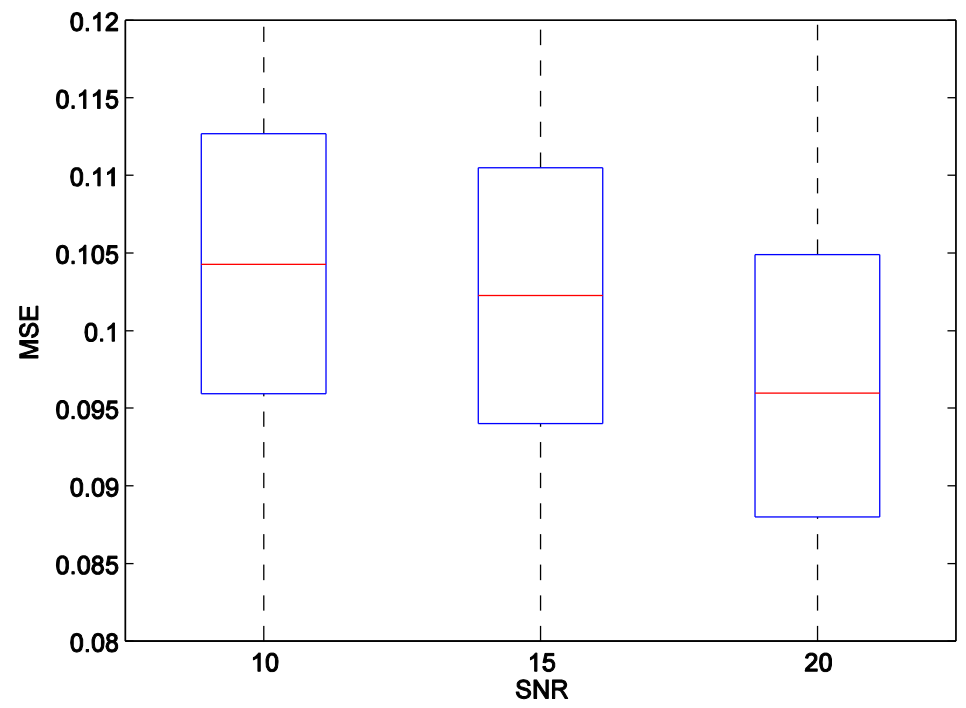

Figure 4.7: Dependence of estimating sensor locations on the signal to noise ratio.

\subsubsection{Sensitivity of the Algorithm to the Choice of Initial Values}

Choosing the initial values of parameters during their numerical evaluation has a significant effect on the the performance of the bisection algorithm. Therefore, in this section we are going to study this effect. We will partition the area of interest $A$ into four no overlapping rings with the true location of the target and the sensor as the center of the rings. The radii of the rings are increasing from one to four, and the initial values are randomly chosen within each ring. The first ring is the closest to the true location and the fourth ring is the farthest one. As seen from Fig. 4.8, the closer we are to the true location of sensor and target the more accurate our results are. 


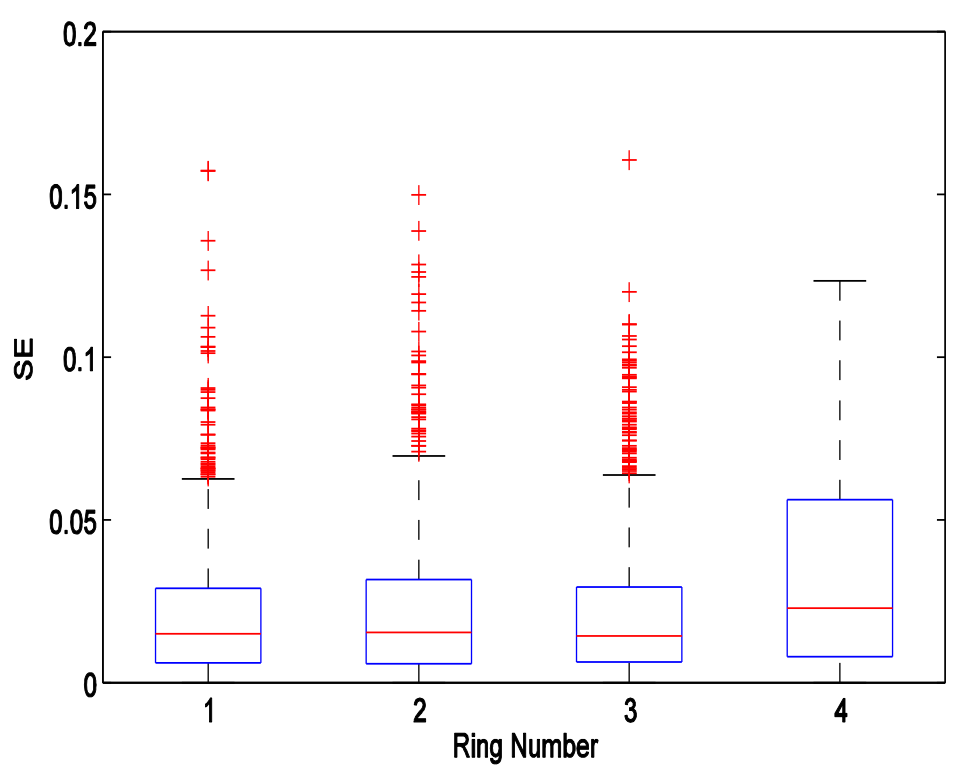

Figure 4.8: The effect of choosing the initial values on the performance of the estimator.

\subsection{Numerical Results for Digital Case}

In this section we evaluate performance of the proposed algorithm when the system is sending quantized data to the FC. The performance is displayed as a function of few parameters such as the number of sensors $\mathrm{K}$, the number of quantization levels $M$, the number of estimated sensor location measurements per sensor, and SNRs. It is measured in terms of square error (SE) and in terms of probability of outliers.

The assumed area is $10 \times 10$, the number of quantization levels is $M=8, S N R_{o}=20$, $\xi^{2}=0.4, \sigma^{2}=15.6782$, the target location $x_{c}=y_{c}=5, S N R_{c}=20, \eta^{2}=0.1280$. All results are obtained using 1000 Monte Carlo simulations.

\subsubsection{The Effect of Varying the Number of Sensors}

In this experiment we evaluate how the number of sensors deployed over the area affects the performance of the developed estimator. We fix almost all parameters in this experiment, but the number of sensors. The number of sensors is varied as follows: 10, $20,30,50,70,90$. The number of estimated sensor location measurements per sensor is fixed at $N=5$. The number of quantization levels is fixed at $M=8$. After performing 
this experiment we noticed that with the increasing the number of sensors over the area the value of the square error is decreasing, as expected. We also observe from Fig. 4.9 that increasing the number of sensors from $K=10$ to $K=20$ has significant effect on the performance of the estimator.

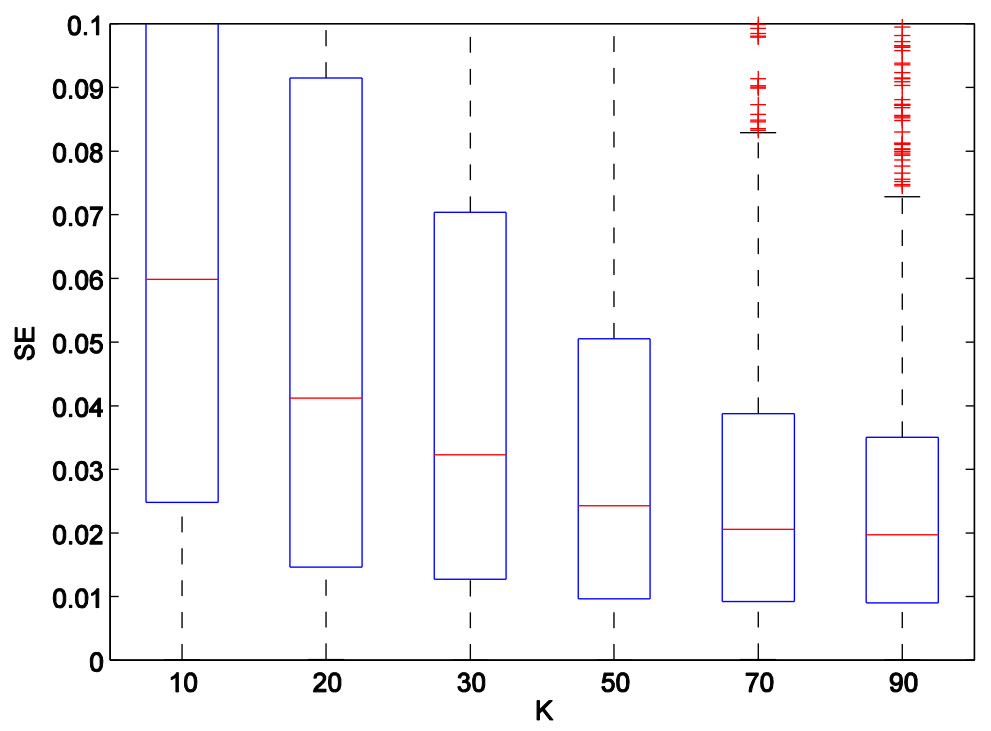

Figure 4.9: Dependence of the simulated SE on the number of sensors for the case of the digital channel with $M=8$.

\subsubsection{Varying the Strength of the Field $\Lambda$}

In this experiment we show how the strength of the field can influence the performance of the designed estimator in the digital case. The number of sensors is fixed at $K=50$ and the number of estimated sensor location measurements per sensor is fixed at $N=5$. The number of quantization levels is fixed at $M=8$ and the field strength $\Lambda$ is varied as $10,30,50,70,90,100$. Since the strength of the field has major effect on the signal to noise ratio, it is expected that increasing the strength of the field will improve the performance of the designed estimator. As seen in Fig. 4.10, this hypothesis is confirmed. We also observe that when the strength of the field is above $70, \Lambda>70$, varying the strength of the field has a minor effect on the performance of the estimator. 


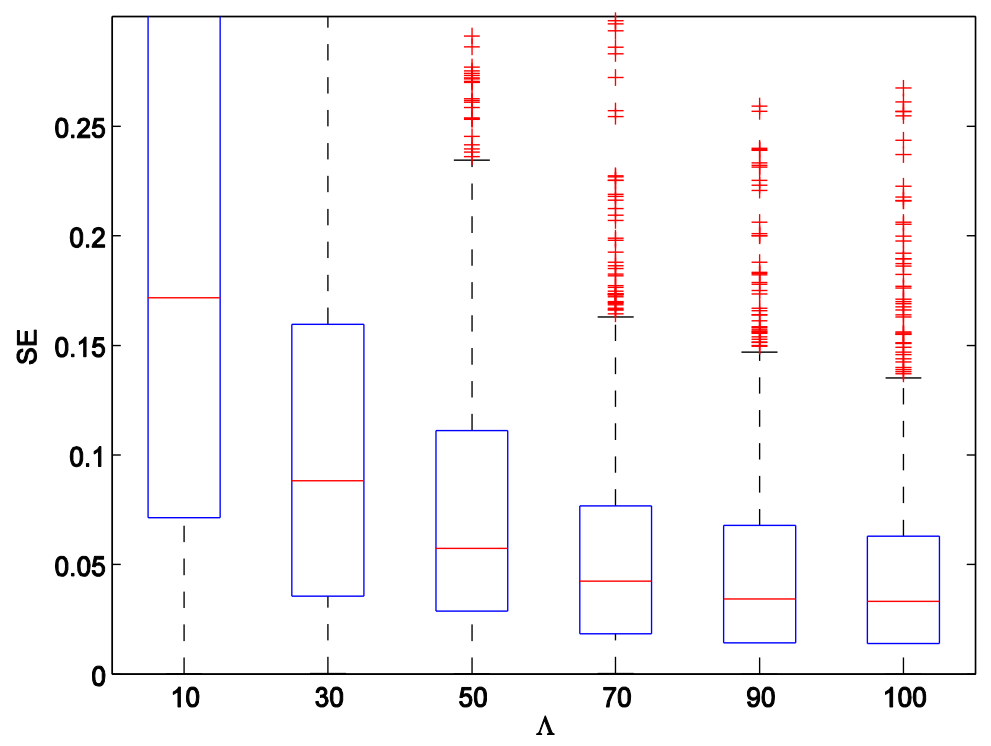

Figure 4.10: Dependence of the simulated SE on the strength of the field in the case of the digital channel with $M=8$.

\subsubsection{Probability of Outliers with Varying the Number of Sensors}

To take a closer look at the performance of the proposed estimator, we evaluated the probability of outliers for different values of $K=10,30,70$. To obtain the plots shown in Fig. 4.11, we vary the value of the threshold $\tau$ in the interval $[0,1]$ with the step 0.1 .

From the generated plots, as anticipated, the probability of outliers decreases when the threshold value $\tau$ increases. We notice as well that with increasing the number of sensors the probability of outliers is decreasing, as expected.

\subsubsection{Comparison with the Case when Sensor Locations are known}

Similar to the case considered in Sec.4.2.5, we compare the performance of our distributed estimator with a baseline performance. We assume that the baseline case estimates only location of the object, while the sensor locations are known. Fig. 4.12 shows the plot of the square error with two different estimators. In this experiment the number of sensors $\mathrm{K}$ is set to 50 . The SNR in observation and transmission channel is set to $S N R_{o}=S N R_{c}=20$. 


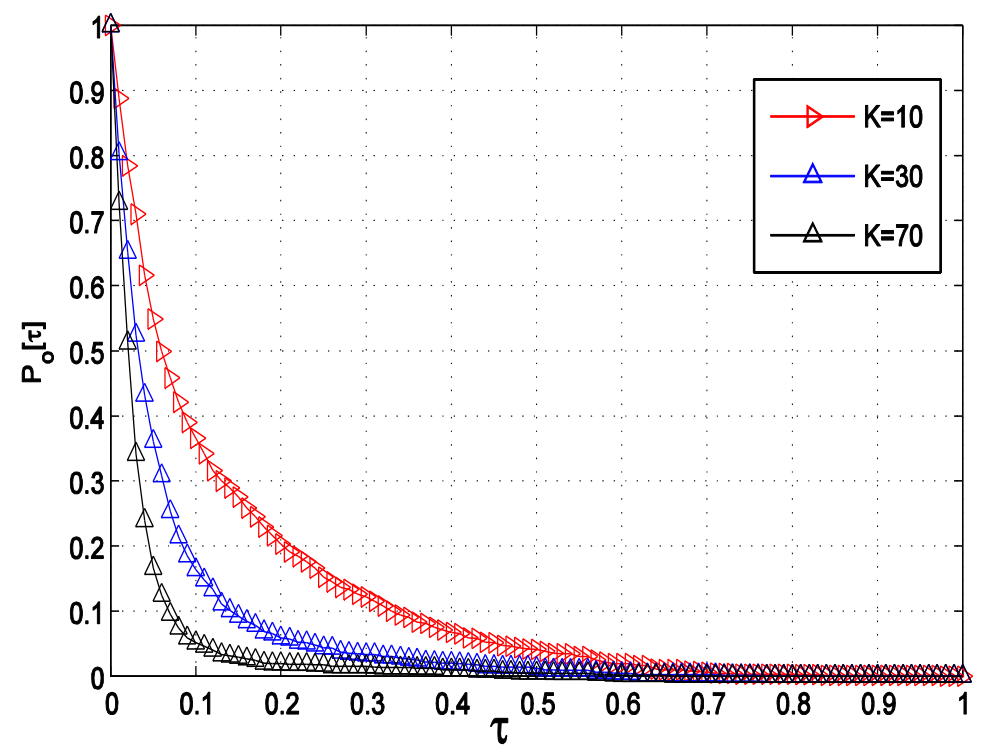

Figure 4.11: Dependence of the probability of outliers on the threshold $\tau$ for three choices of $K, K=10,30$, and 70 .

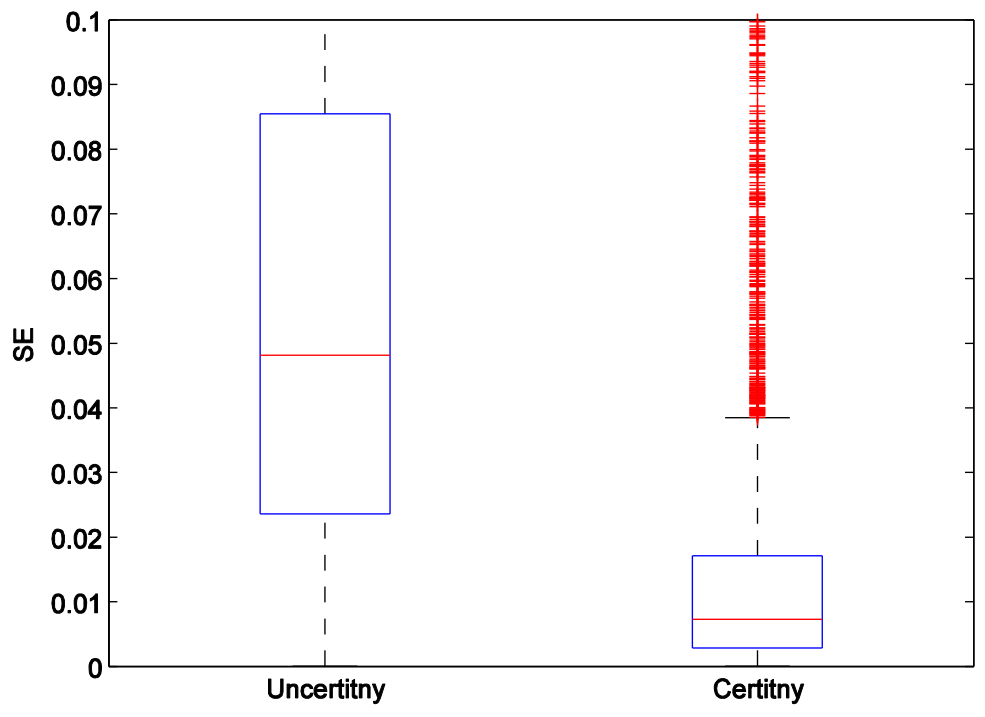

Figure 4.12: The performance comparison between estimators with certainty and uncertainty in sensor locations. The network is composed of $K=50$ sensors.

\subsubsection{Varying Signal to Noise Ratio}

In this section we vary the value of the signal to noise ratio and evaluate its effect on the performance of the proposed algorithm. The number of sensors is fixed at $K=50$, 
the number of estimated sensor location measurements per sensor is set to $N=5$, and the number of quantization levels is set to $M=8$. In order to perform this simulation, the signal to noise ratio in the observation channel $S N R_{o}$ and the signal to noise ratio $S N R_{c}$ in the transmission channel each takes values $10,15,20$. The noise variance in the observation and transmission channels is calculated for each value of the signal to noise ratio. From the generated box plot shown in Fig. 4.13, as expected, the square error of target estimation is decreasing with increasing signal to noise ratios.

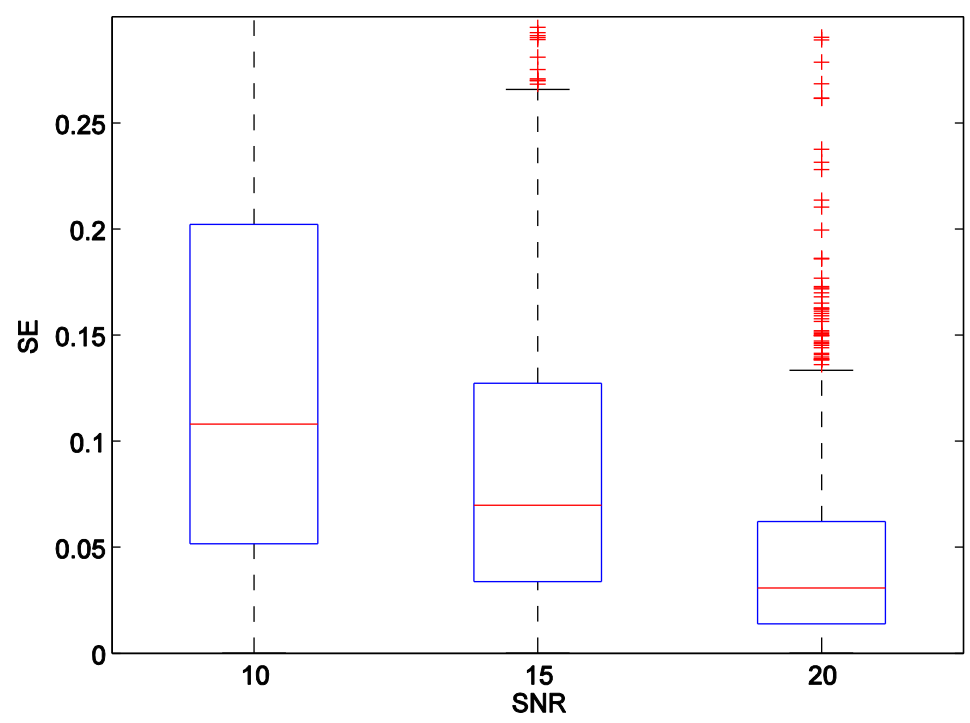

Figure 4.13: Dependence of the simulated SE on the signal to noise ratio, $S N R_{o}=S N R_{c}$.

We also evaluated the probability of outliers for the same values of the signal to noise ratio. Three plots parametrized by three different SNR values are shown in Fig. 4.14. 


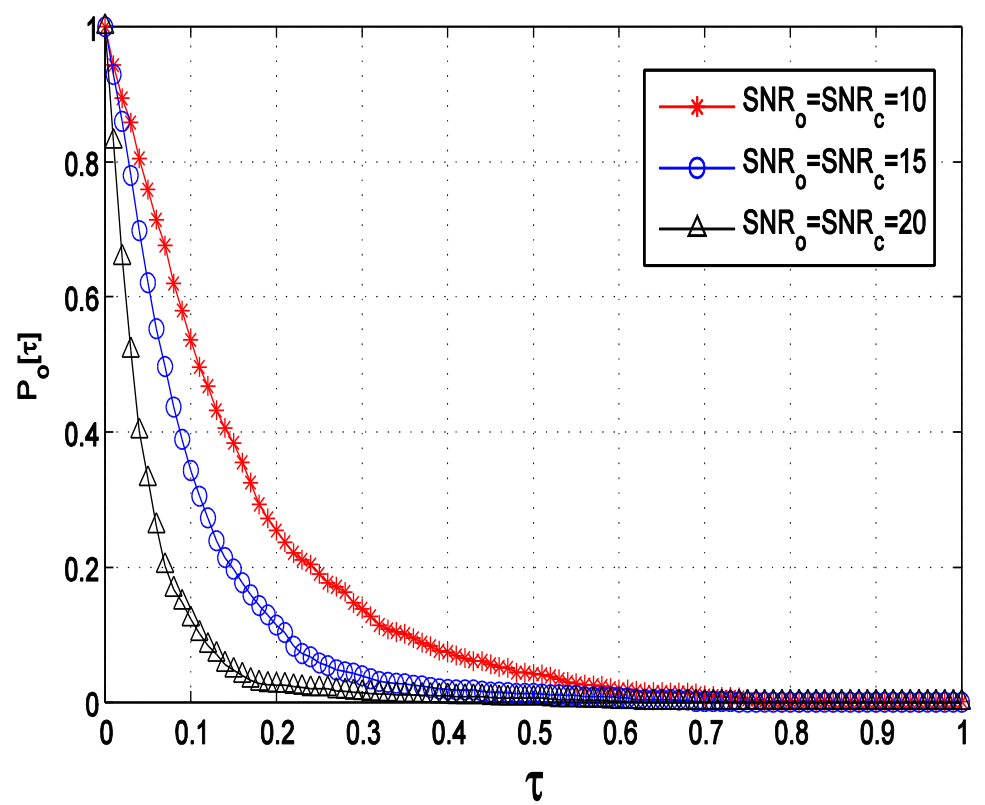

Figure 4.14: Dependence of the probability of outliers on the threshold values. The plots are parametrized by three different values of $S N R_{o}=S N R_{c}=10,15,20$.

As well, in Fig. 4.15 we also observe the effect of varying SNR on the performance of estimating sensor locations.

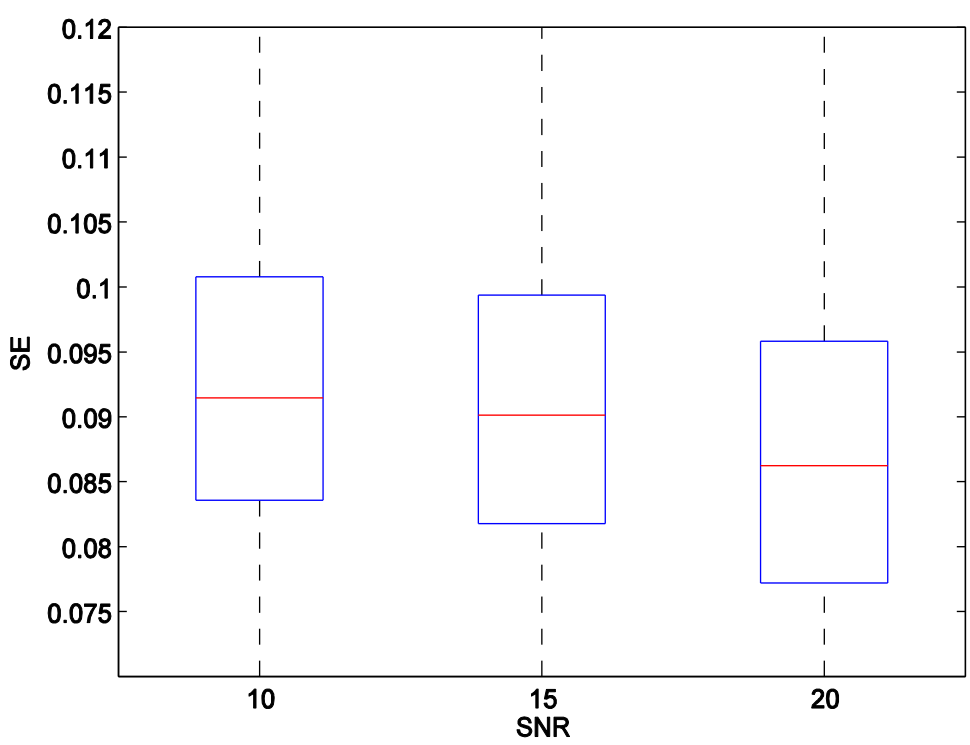

Figure 4.15: Dependence of estimating sensor locations on the signal to noise ratio. 


\subsubsection{Varying the Number of Quantization Levels}

Quantization is an important factor in the process of the digital modulation. In this experiment we will evaluate the effect of this factor on the performance of the distributed estimator by varying the number of quantization levels.

To conduct this experiment, we start with the number of quantization levels $M=4$ and we implement 500 Monte Carlo simulations. Then, we do the same for $M=8, M=16$, $M=32$ and $M=128$. Since increasing the number of quantization levels will reduce quantization error, the performance of the estimator improves with increasing the number of quantization levels. We also note that, when $M=128$ the performance of the digital case converges to the performance of the analog case. This result is displayed in Fig. 4.16.

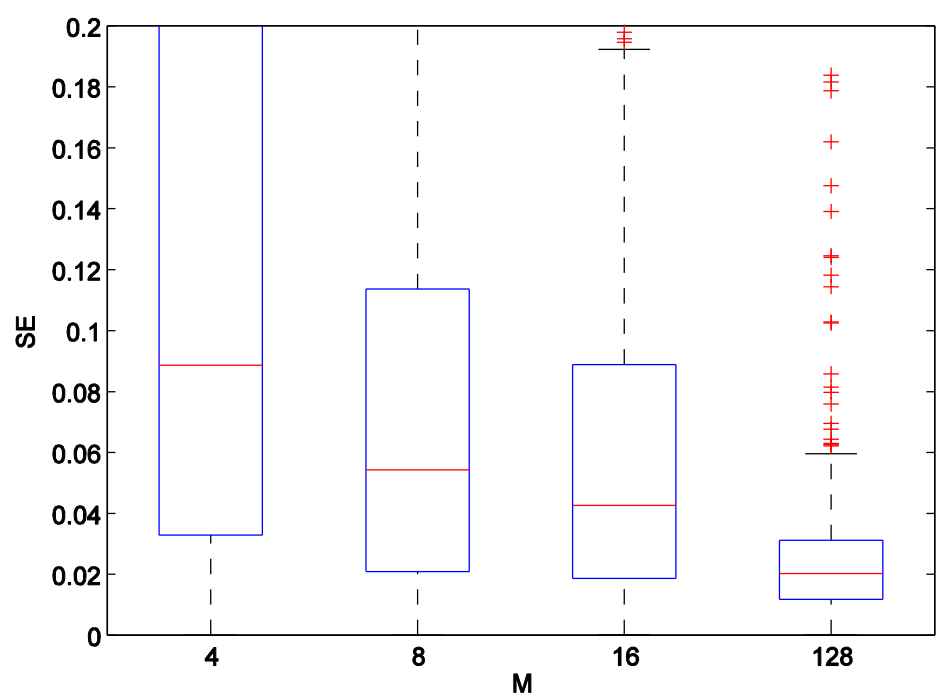

Figure 4.16: Dependence of the simulated SE on the Number of Quantization Levels.

\subsubsection{Sensitivity of the Algorithm to the Choice of Initial Values}

Similarly, what did in the analog case we are going to study the the sensitivity of our algorithm to the choice of initial values. We will partition the area of interest $A$ into four no overlapping rings with the true location of the target and the sensor as the center of 
the rings. The radii of the rings are increasing from one to four, and the initial values are randomly chosen within each ring. The first ring is the closest to the true location and the fourth ring is the farthest one. From Fig. 4.17, we observe that closest ring to the true location has the best performance compared to the other rings.

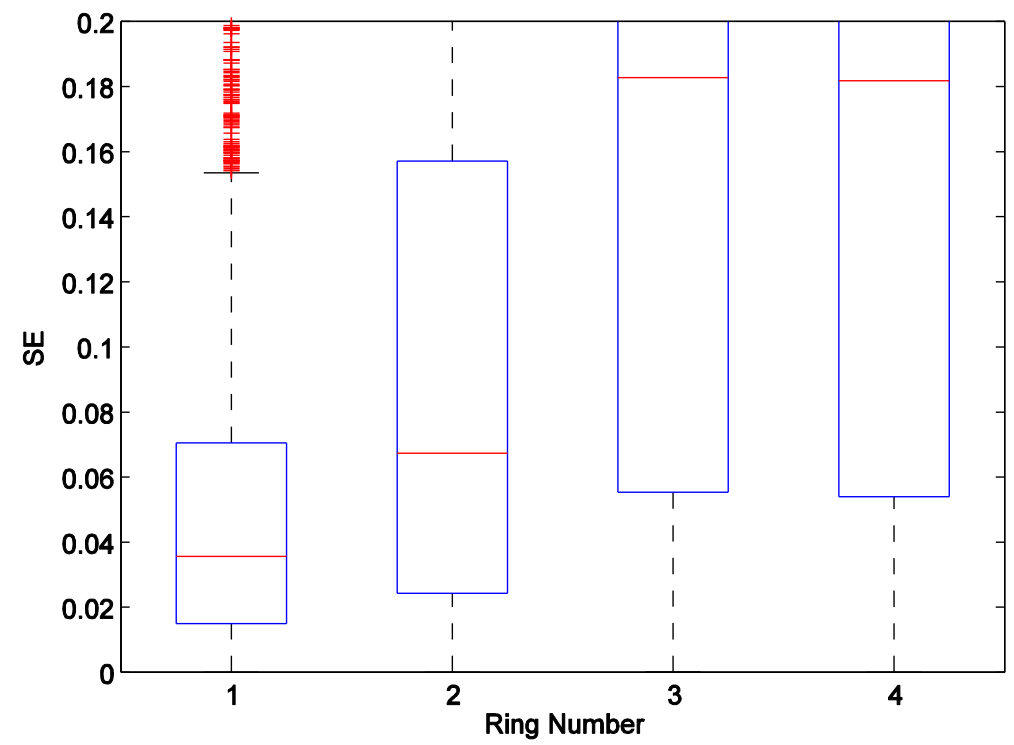

Figure 4.17: The effect of choosing the initial values on the performance of the estimator. 


\section{Chapter 5}

\section{Conclusion and Future Work}

\subsection{Summary}

In this thesis we studied the problem of distributed joint sensor and target locations estimation. The problem can be summarized as following. We have an area $10 \times 10$. Sensors are randomly distributed over this area. There is a source point within this area generating a deterministic parametric field. The location of the source point and the true positions of sensors are unknown, but we have noisy measurements of the sensor locations via positioning technology system such as GPS. The noisy measurements are realizations of Gaussian random variables with the true locations of the sensors as their mean and known variance. The sensors are measuring the field. There is an AWGN added to these measurements due to observation channel. The data observed by sensors are sent to the FC through AWGN channels.

We considered two cases of transmitting data to the FC. The first case assumes the transmission of raw data and uses a linear analog modulation (such as Amplitude modulation), the second case assumes that the data are quantized and uses a linear digital modulation (such as ON-OFF keying). At the FC we applied MLE solution in order to estimate the unknown parameters of the field and the true sensor locations. Finally, we used the bisection algorithm as our iterative method to solve the highly nonlinear equations representing MLE solution.

Based on the numerical results generated and discussed in chapter four, we conclude 
that the performance of the proposed algorithm in the case of analog modulation is better than the one in the case of digital modulation due to the quantization error in the digital case. In addition to that, the performance is effected by different parameters such as the density of the distributed sensors over the area, signal to noise ratio, the strength of the field, and the number of quantization levels in the case of digital modulation. These effects can be observed mainly when there are few sensors distributed over the area.

To summarize the overall results, the algorithm is robust and converging with few number of sensors, as well as the performance is improving and the number of outliers decreasing with the increasing number of sensors, the strength of the field, the signal to noise ratio, and with the increasing number of quantization levels in the case of digital modulation.

\subsection{Claimed Novelties}

This work claims the following novelties:

- Formulating the problem and deriving all equations including the Likelihood function and applying the MLE estimator in order to estimate the unknown parameters of the true sensor locations and the target location.

- The robustness of the bisection algorithm. This algorithm was applied to solve highly nonlinear equations representing the MLE solution.

- The justification of Gaussian noise models due to linear analog and digital modulations for data transmission.

- The performance analysis of the proposed solution.

\subsection{Future Research}

In the future, this work can be extended in many different ways. The suggested future work can be summarized as follows: 
- We considered the shape of the field to be Gaussian bell function. Other shapes, such as field decays according to the inverse square law, can be used.

- In this thesis the FC is collecting data from sensors and estimating the location of the target. Other approaches can assume that sensors are communicating with each other to define the location of the target. We can also assume that the target is moving.

- The source point in our case is assumed to be a single target fixed at a certain point. In the future we can assume a mluti-target scenario.

- The type of WSN in our case is considered to be a homogeneous network. In the future we can consider a heterogeneous network, where each sensor is characterized by a different noise.

- The communication channel is assumed in our case to be AWGN channel. This can be changed to include fading effects. 


\section{References}

[1] D. E. Culler and W. Hong, "Wireless sensor networks.," Communications of the ACM, vol. 47, no. 6, pp. $30-33,2004$.

[2] E. Gaura, Wireless Sensor Networks: Deployments and Design Frameworks, Springer, Coventry, 2010.

[3] E. H Callaway, Wireless sensor networks: architectures and protocols, vol. 3, CRC press, Boka Raton, 2004.

[4] I. F. Akyildiz, W. Su, Y. Sankarasubramaniam, and E. Cayirci, "Wireless sensor networks: a survey," Computer Networks, vol. 38, no. 4, pp. 393 - 422, 2002.

[5] K. Sohraby, D. 1. Minoli, and T. Znati, Wireless sensor networks: technology, protocols, and applications, Wiley-interscience, New York, 2007.

[6] M. Ibnkahla, Wireless Sensor Networks: A Cognitive Perspective, vol. 3, CRC Press, Boca Raton, 2012.

[7] G. Mao and B. Fidan, Localization algorithms and strategies for wireless sensor networks, Information Science Reference-Imprint of: IGI Publishing, Hershey, 2009.

[8] C. S. Raghavendra, K. M. Sivalingam, and T. Znati, Wireless sensor networks, Kluwer Academic Pub, New York, 2004.

[9] D. Dardari and F. Fabbri, "Spatial field estimation through wireless sensor networks under bandwidth constraints," in Global Telecommunications Conference (GLOBECOM 2010), 2010 IEEE, Miami, FL, 2010, pp. 1-5.

[10] Lin Fang and R. J. P. De Figueiredo, "Energy-efficient scheduling optimization in wireless sensor networks with delay constraints," in Communications, 2007. ICC '07. IEEE International Conference on, 2007, pp. 3734-3739.

[11] W. Dargie and C. Poellabauer, Fundamentals of wireless sensor networks: theory and practice, Wiley, West Sussex, 2010.

[12] N. Sharma, M. K. Singh, and A. Goswami, "A heuristic approach for component selection of low power micro sensor nodes based on simple energy model," in Electronics Computer Technology (ICECT), 2011 3rd International Conference on, 2011, vol. 4, pp. 290-295. 
[13] L. Cui, F. Wang, H. Luo, H. Ju, and T. Li, “A pervasive sensor node architecture," in Network and Parallel computing, pp. 565-567. Springer, Beijing, 2004.

[14] F. L. Lewis, “Wireless sensor networks," Smart environments: technologies, protocols, and applications, pp. 1-18, 2004.

[15] F. Hu and X. Cao, Wireless sensor networks: principles and practice, Auerbach Publications, Tamil Nadu, 2010.

[16] J. Li, Distributed estimation in resource-constrained wireless sensor networks, Ph.D. thesis, Georgia Institute of Technology, 2008.

[17] A. Pal, "Localization algorithms in wireless sensor networks: Current approaches and future challenges," Network Protocols and Algorithms, vol. 2, no. 1, pp. 45-73, 2010.

[18] X. Ji, Localization algorithms for wireless sensor network systems, Ph.D. thesis, The Pennsylvania State University, 2004.

[19] B. Cheng, R. Du, B. Yang, W. Yu, C. Chen, and X. Guan, “An accurate gps-based localization in wireless sensor networks: a gm-wls method," in Parallel Processing Workshops (ICPPW), 2011 40th International Conference on. IEEE, 2011, pp. 33-41.

[20] M. Terwilliger, Localization in wireless sensor networks, Ph.D. thesis, Western Michigan University, 2006.

[21] X. Liu, E. Zhao, and K. Chen, "Energy efficient beacon discovering scheme for node localization in wireless sensor networks," Wuhan University Journal of Natural Sciences, vol. 15, no. 4, pp. 303-307, 2010.

[22] A. Perrig, J. Stankovic, and D. Wagner, "Security in wireless sensor networks.," Communications of the ACM, vol. 47, no. 6, pp. 53 - 57, 2004.

[23] J. Yick, B. Mukherjee, and D. Ghosal, "Wireless sensor network survey," Computer Networks, vol. 52, no. 12, pp. 2292 - 2330, 2008.

[24] G. J. Pottie and W. J. Kaiser, "Wireless integrated network sensors.," Communications of the ACM, vol. 43, no. 5, pp. $51-58,2000$.

[25] I. F. Akyildiz, T. Melodia, and K. R. Chowdhury, “Wireless multimedia sensor networks: Applications and testbeds," Proceedings of the IEEE, vol. 96, no. 10, pp. 15881605, 2008.

[26] A. Boukerche, H. A. B. Oliveira, E. F. Nakamura, and A. A. F. Loureiro, "Localization systems for wireless sensor networks," IEEE Trans. on Wireless Communications, vol. 14 , no. 6, pp. 6-12, 2007.

[27] J. Kenyeres, M. Kenyeres, M. Rupp, and P. Farkas, “Wsn implementation of the average consensus algorithm," in Wireless Conference 2011 - Sustainable Wireless Technologies (European Wireless), 11th European, 2011, pp. 1-8. 
[28] S. S. Pereira, S. Barbarossa, and A. Pages-Zamora, "Consensus for distributed embased clustering in wsns," in Sensor Array and Multichannel Signal Processing Workshop (SAM), 2010 IEEE, 2010, pp. 45-48.

[29] J. Choi, S. Li, X. Wang, and J. Ha, "A general distributed consensus algorithm for wireless sensor networks," in Wireless Advanced (WiAd), 2012, 2012, pp. 16-21.

[30] J. Xiao and Z. Luo, “Decentralized estimation in an inhomogeneous sensing environment," Information Theory, IEEE Transactions on, vol. 51, no. 10, pp. 3564-3575, 2005.

[31] J. Y. Wu, Q. Z. Huang, and T. S. Lee, "Energy-constrained decentralized best-linearunbiased estimation via partial sensor noise variance knowledge," Signal Processing Letters, IEEE, vol. 15, pp. 33-36, 2008.

[32] S. Cui, J. Xiao, A. J. Goldsmith, Z. Luo, and H. V. Poor, “Estimation diversity and energy efficiency in distributed sensing," Signal Processing, IEEE Transactions on, vol. 55, no. 9, pp. 4683-4695, 2007.

[33] J. Li and G. AlRegib, “Distributed estimation in energy-constrained wireless sensor networks," Signal Processing, IEEE Transactions on, vol. 57, no. 10, pp. 3746-3758, 2009.

[34] J. Li and G. AlRegib, "Function-based network lifetime for estimation in wireless sensor networks," Signal Processing Letters, IEEE, vol. 15, pp. 533-536, 2008.

[35] Y. Wang, P. Ishwar, and V. Saligrama, "One-bit distributed sensing and coding for field estimation in sensor networks," Signal Processing, IEEE Transactions on, vol. 56, no. 9, pp. 4433-4445, 2008.

[36] A. Ribeiro and G. B. Giannakis, "Bandwidth-constrained distributed estimation for wireless sensor networks-part i: Gaussian case," Signal Processing, IEEE Transactions on, vol. 54, no. 3, pp. 1131-1143, 2006.

[37] R. Niu, B. Chen, and P. K. Varshney, "Fusion of decisions transmitted over rayleigh fading channels in wireless sensor networks," Signal Processing, IEEE Transactions on, vol. 54, no. 3, pp. 1018-1027, 2006.

[38] R. Niu and P. K. Varshney, "Target location estimation in sensor networks with quantized data," Signal Processing, IEEE Transactions on, vol. 54, no. 12, pp. 4519-4528, 2006.

[39] P. Addesso, S. Marano, and V. Matta, "Estimation of target location via likelihood approximation in sensor networks," Signal Processing, IEEE Transactions on, vol. 58, no. 3, pp. 1358-1368, 2010.

[40] A. Gersho and R. M. Gray, Vector quantization and signal compression, vol. 159, Kluwer Academic Pub, Santa Barbara, 1992. 
[41] I. J. Myung, “Tutorial on maximum likelihood estimation," Journal of Mathematical Psychology, vol. 47, no. 1, pp. 90 - 100, 2003.

[42] H. L. Van Trees, Detection, Estimation, and Modulation Theory: Vol. I, Wiley Interscience, New York, 2001.

[43] J. Daintith and R. D. Nelson, The Penguin dictionary of mathematics, Penguin Group, London, 1989.

[44] G. R. Lindfield and J. J. E. Penny, Numerical Methods: Using MATLAB, Academic Press, Waltham, 2012.

[45] B. Hahn and D. Valentine, Essential MATLAB for engineers and scientists, Newnes, Burlington, 2007. 